CRYSTALLOGRAPHIC COMMUNICATIONS

ISSN 2056-9890

Received 11 August 2017

Accepted 11 October 2017

Edited by S. Parkin, University of Kentucky, USA

Keywords: crystal structure; purpurin; anthraquinone; hydrogen bonding.

CCDC references: $1579381 ; 1579380$

Supporting information: this article has supporting information at journals.iucr.org/e

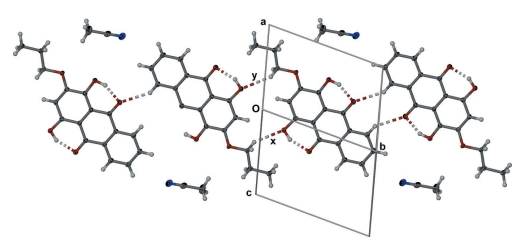

OPEN Ә ACCESS

\section{Synthesis and crystal structures of two purpurin derivatives: 1,4-dihydroxy-2-propoxyanthra- quinone and 2-butoxy-1,4-dihydroxyanthraquinone}

\author{
Eric Bosch* and Emily N. McClain
}

Department of Chemistry, Missouri State University, Springfield, MO 65897, USA. *Correspondence e-mail: ericbosch@missouristate.edu

The title compounds were obtained by deprotonation of 1,2,4-trihydroxyanthraquinone (purpurin) using sodium hydride followed by reaction with either 1-bromopropane or 1-bromobutane. 1,4-Dihydroxy-2-propoxyanthraquinone crystallizes as a 1:1 solvate from acetonitrile, $\mathrm{C}_{17} \mathrm{H}_{14} \mathrm{O}_{5} \cdot \mathrm{CH}_{3} \mathrm{CN}$. The anthraquinone core of the molecule is essentially planar and both hydroxy groups participate in intramolecular $\mathrm{O}-\mathrm{H} \cdots \mathrm{O}$ (carbonyl) hydrogen bonds. The propyl chain is angled slightly above the plane of the anthraquinone moiety with a maximum deviation of 0.247 (2) $\AA$ above the plane for the terminal carbon atom. In contrast, 2-butoxy-1,4-dihydroxyanthraquinone, $\mathrm{C}_{18} \mathrm{H}_{16} \mathrm{O}_{5}$, crystallizes from nitromethane with two independent molecules in the asymmetric unit. The anthraquinone core of each independent molecule is essentially planar and both hydroxy groups on both molecules participate in intramolecular $\mathrm{O}-$ $\mathrm{H}$. . O (carbonyl) hydrogen bonds. The butyl chain in one molecule is also angled slightly above the plane of the anthraquinone moiety, with a maximum deviation of 0.833 (5) $\AA$ above the plane for the terminal carbon atom. In contrast, the butyl group on the second molecule is twisted out of the plane of the anthraquinone core with a torsion angle of $65.1(3)^{\circ}$, resulting in a maximum deviation of 1.631 (5) $\AA$ above the plane for the terminal carbon atom.

\section{Chemical context}

Purpurin, 1,2,4-trihydroxy anthraquinone, is a major component of the dye extracted from madder root (Schweppe \& Winter, 1997). The extract from madder root has been used to dye wool and other fabrics since antiquity. Purpurin is commercially available and we here report two derivatives, 1,4-dihydroxy-2-propoxy anthraquinone and 2-butoxy-1,4dihydroxy anthraquinone, prepared by selective deprotonation of purpurin followed by alkylation with the either 1-bromopropane or 1-bromobutane.<smiles></smiles>

(1)<smiles>CCCCOc1cc2c3c(c1O[Hg]O3)C(=O)c1ccccc1C2=O</smiles>

(2) 
Table 1

Hydrogen-bond geometry $\left(\AA,^{\circ}\right)$ for $(1)$.

\begin{tabular}{|c|c|c|c|c|}
\hline$D-\mathrm{H} \cdots A$ & $D-\mathrm{H}$ & $\mathrm{H} \cdots A$ & $D \cdots A$ & $D-\mathrm{H} \cdots A$ \\
\hline $\mathrm{O} 1-\mathrm{H} 1 O \cdots \mathrm{O} 5$ & $0.88(1)$ & $1.75(1)$ & $2.5537(13)$ & $152(2)$ \\
\hline $\mathrm{O} 3-\mathrm{H} 3 \mathrm{O} \cdots \mathrm{O} 4$ & $0.87(1)$ & $1.75(1)$ & 2.5578 (13) & $153(2)$ \\
\hline $\mathrm{C} 10-\mathrm{H} 10 \cdots \mathrm{N} 1$ & 0.95 & 2.73 & 3.4009 (19) & 128 \\
\hline $\mathrm{C} 15-\mathrm{H} 15 A \cdots \mathrm{O} 3^{\mathrm{i}}$ & 0.99 & 2.57 & 3.2179 (16) & 123 \\
\hline $\mathrm{C} 11-\mathrm{H} 11 \cdots \mathrm{O}^{\mathrm{ii}}$ & 0.95 & 2.47 & $3.2446(17)$ & 138 \\
\hline
\end{tabular}

Symmetry codes: (i) $-x+1,-y,-z+1$; (ii) $-x+2,-y+2,-z+1$.

\section{Structural commentary}

The asymmetric unit of 1,4-dihydroxy-2-propoxy anthraquinone (1), crystallized from acetonitrile solvent, contains a single anthraquinone molecule and one acetonitrile solvate molecule as shown in Fig. 1. The two intramolecular hydrogen bonds (Table 1) are typical for the 1,4-dihydroxy anthraquinones and 1-hydroxyanthraquinones. These hydrogen bonds are maintained in chloroform solution, as shown by the chemical shift of 13.47 and $13.56 \mathrm{ppm}$ for the two hydroxyl protons. The anthraquinone moiety is planar, with an average root mean square (r.m.s.) deviation of atoms $\mathrm{C} 1$ to $\mathrm{C} 14$ of $0.021 \AA$, in which the maximum deviation from the plane defined by atoms $\mathrm{C} 1$ to $\mathrm{C} 14$ is 0.044 (1) $\AA$ for C9. The propyl chain is angled slightly above the plane of the anthraquinone moiety, with deviations of 0.043 (2), 0.143 (2) and 0.247 (2) $\AA$ for atoms $\mathrm{C} 15, \mathrm{C} 16$ and $\mathrm{C} 17$, respectively, from the plane defined by atoms $\mathrm{C} 1-\mathrm{C} 14$. The acetonitrile is angled towards H10 with a N1‥C10 distance of 3.401 (2) A. The final difference map shows several peaks of 0.2 to $0.7 \mathrm{e}^{-3}$ in the anthraquinone plane that suggest the presence of minor whole-molecule disorder in which the anthraquinone is translated in the plane and/or flipped over.

In contrast, the asymmetric unit of 2-butoxy-1,4-dihydroxy anthraquinone (2) crystallized from nitromethane solvent, contains two unique anthraquinone molecules as shown in Fig. 2. Both molecules feature two intramolecular hydrogen bonds (Table 2) similar to those observed in (1). These hydrogen bonds are also maintained in chloroform solution, as shown by the chemical shift of 13.46 and $13.55 \mathrm{ppm}$ for the two hydroxyl protons. The anthraquinone moieties in both molecules are planar. The r.m.s deviation of atoms $\mathrm{C} 1$ to $\mathrm{C} 14$ is $0.006 \AA$, with a maximum deviation from the plane defined by atoms $\mathrm{C} 1$ to $\mathrm{C} 14$ of 0.011 (2) $\AA$ for C13. The r.m.s. deviation

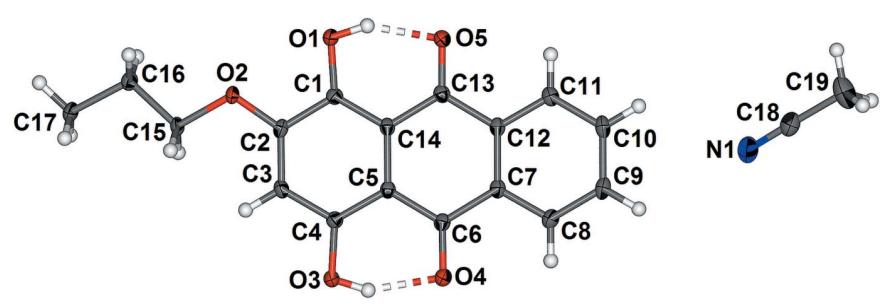

Figure 1

Molecular structure of (1) with the included acetonitrile. Displacement ellipsoids of non- $\mathrm{H}$ atoms are drawn at the $50 \%$ probability level and $\mathrm{H}$ atoms are shown as circles of arbitrary size.
Table 2

Hydrogen-bond geometry $\left(\AA,^{\circ}\right)$ for (2).

\begin{tabular}{|c|c|c|c|c|}
\hline$D-\mathrm{H} \cdots A$ & $D-\mathrm{H}$ & $\mathrm{H} \cdots A$ & $D \cdots A$ & $D-\mathrm{H} \cdots A$ \\
\hline $\mathrm{O} 1-\mathrm{H} 1 O \cdots \mathrm{O} 5$ & $0.85(2)$ & $1.78(2)$ & $2.564(3)$ & $153(3)$ \\
\hline $\mathrm{O} 3-\mathrm{H} 3 \mathrm{O} \cdots \mathrm{O} 4$ & $0.87(2)$ & $1.74(2)$ & $2.536(3)$ & $151(3)$ \\
\hline $\mathrm{O} 6-\mathrm{H} 6 \mathrm{O} \cdots \mathrm{O} 10$ & $0.86(2)$ & $1.72(2)$ & $2.542(3)$ & $158(3)$ \\
\hline $\mathrm{O} 8-\mathrm{H} 8 \mathrm{O} \cdots \mathrm{O} 9$ & $0.87(2)$ & $1.73(2)$ & $2.554(3)$ & $155(3)$ \\
\hline $\mathrm{C} 3-\mathrm{H} 3 \cdots \mathrm{O} 10$ & 0.95 & 2.55 & $3.494(3)$ & 173 \\
\hline $\mathrm{C} 29-\mathrm{H} 29 \cdots \mathrm{O} 3$ & 0.95 & 2.41 & $3.231(4)$ & 144 \\
\hline $\mathrm{C} 15-\mathrm{H} 15 B \cdots \mathrm{O} 6^{\mathrm{i}}$ & 0.99 & 2.52 & $3.485(3)$ & 164 \\
\hline $\mathrm{C} 21-\mathrm{H} 21 \cdots \mathrm{O} 8^{\mathrm{ii}}$ & 0.95 & 2.55 & $3.502(3)$ & 180 \\
\hline $\mathrm{C} 33-\mathrm{H} 33 A \cdots \mathrm{O} 9^{\mathrm{iii}}$ & 0.99 & 2.56 & $3.547(4)$ & 172 \\
\hline
\end{tabular}

from the plane defined by atoms $\mathrm{C} 19$ to $\mathrm{C} 32$ is $0.025 \AA$, with a maximum deviation of 0.048 (2) $\AA$ for C31. The butyl chain attached to $\mathrm{O} 2$ is twisted out of the $\mathrm{C} 1-\mathrm{C} 14$ anthraquinone plane with a $\mathrm{O} 2-\mathrm{C} 15-\mathrm{C} 16-\mathrm{C} 17$ torsion angle of $-65.1(3)^{\circ}$. The butyl chain has an anti-conformation, the $\mathrm{C} 15-\mathrm{C} 16-$ C17-C18 torsion angle being $-173.1(2)^{\circ}$. The deviations of the butyl carbon atoms from the anthraquinone plane defined by atoms $\mathrm{C} 1$ to $\mathrm{C} 14$ are 0.101 (4), 0.194 (4), 1.467 (4) and 1.631 (5) $\AA$ for atoms $\mathrm{C} 15, \mathrm{C} 16, \mathrm{C} 17$ and $\mathrm{C} 18$, respectively. The butyl chain in the second unique molecule, attached to $\mathrm{O} 7$, is tilted slightly out of the plane of the anthraquinone with a $\mathrm{C} 20-\mathrm{O} 7-\mathrm{C} 33-\mathrm{C} 34$ torsion angle of $-167.3(2)^{\circ}$. This butyl chain also adopts an anti-conformation, the C33-C34C35-C36 torsion angle being $-175.2(3)^{\circ}$. The resultant deviations of the butyl carbon atoms from the plane defined by atoms $\mathrm{C} 19-\mathrm{C} 32$ are 0.077 (4), $0.428(4), 0.356$ (4) and 0.833 (5) $\AA$ for atoms C33, C34, C35 and C36, respectively. There is a close intermolecular contact between phenyl hydrogen atom $\mathrm{H} 3$ and carbonyl oxygen atom $\mathrm{O} 10$, with a C3...O10 distance of 3.494 (3) $\AA$ (labelled X in Fig. 2). A second close intermolecular contact, between phenyl hydrogen atom $\mathrm{H} 29$ and hydroxyl oxygen atom $\mathrm{O} 3$ gives a C29...O3 distance of 3.231 (4) $\AA$ (labelled Y in Fig. 2).

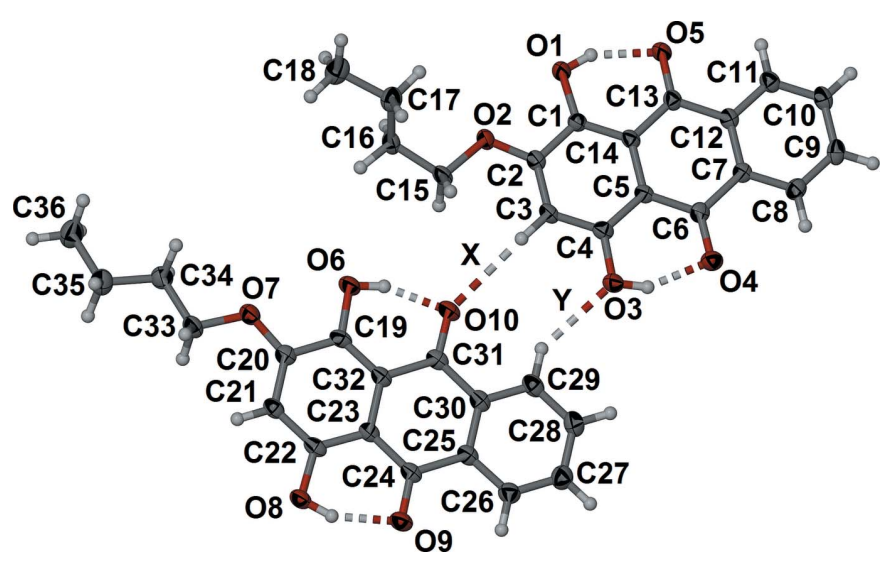

Figure 2

Asymmetric unit of (2) showing the close intermolecular $\mathrm{C}-\mathrm{H} \cdots \mathrm{O}$ contacts $\mathrm{X}$ and $\mathrm{Y}$ (see text). Displacement ellipsoids of non-H atoms are drawn at the $50 \%$ probability level and $\mathrm{H}$ atoms are shown as circles of arbitrary size. 


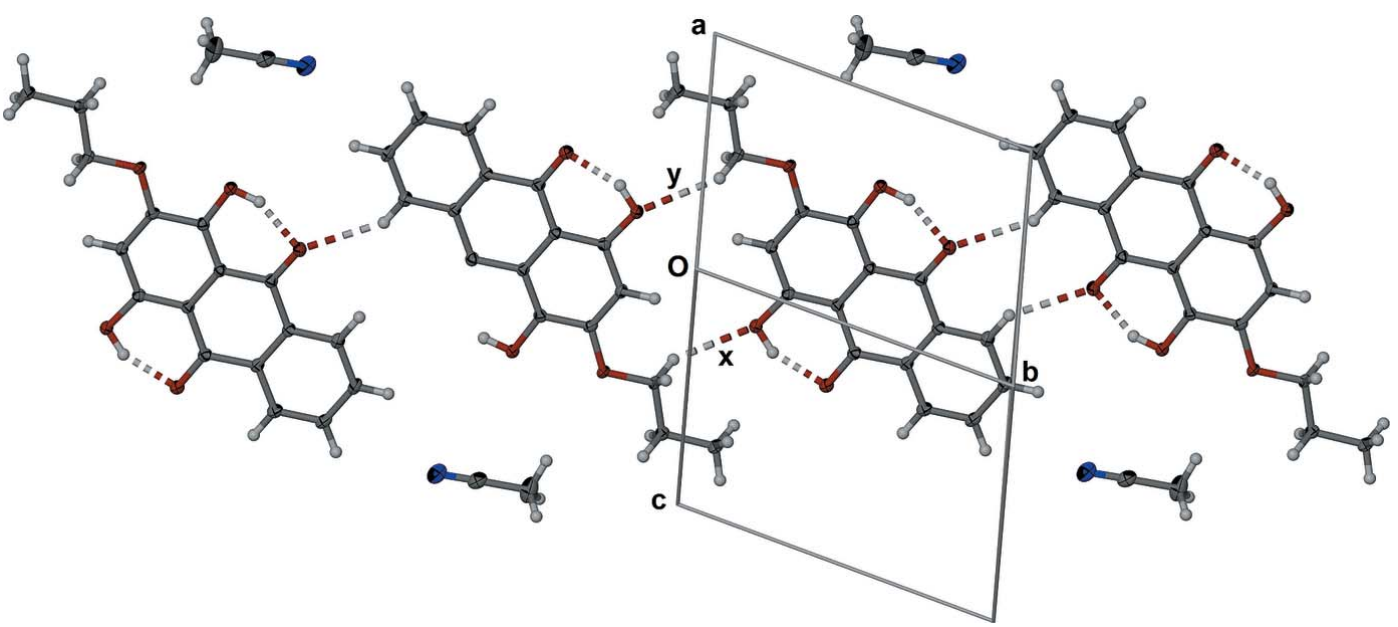

Figure 3

Structure of (1) viewed along the [101] direction, with close $\mathrm{C}-\mathrm{H} \cdots \mathrm{O}$ contacts labelled $\mathrm{x}$ and $\mathrm{y}$ (see text).

\section{Supramolecular features}

In the crystal, molecules of (1) form planes that incorporate the acetonitrile molecule, as shown in Fig. 3. The acetonitrile molecule is almost coplanar with the anthraquinone moiety, with deviations of 0.401 (2), $0.536(2)$ and $0.722(2) \AA$ for atoms $\mathrm{N} 1, \mathrm{C} 18$, and $\mathrm{C} 19$, respectively, from the plane defined by atoms $\mathrm{C} 1-\mathrm{C} 14$. There is a close $\mathrm{C}-\mathrm{H} \cdots \mathrm{O}$ interaction (Table 1) between a phenyl hydrogen atom and an adjacent carbonyl oxygen atom of an inversion-related molecule of (1). The C11 …O5 \#2 distance is 3.245 (2) $\AA$ [symmetry code: (\#2) $2-x, 2-y, 1-z]$ and the interaction is labelled $\mathrm{x}$ in Fig. 3 . The methylene hydrogen $\mathrm{H} 15 \mathrm{~A}$ is close to the carbonyl oxygen $\mathrm{O} 3$ of a second inversion-related molecule of (1). The C15 ... O3\#1 distance is 3.218 (2) $\AA$ [symmetry code: (\#1) $1-x$, $-y, 1-z]$, and the interaction is labelled y in Fig. 3. The anthraquinone units of (1) alternately $\pi$-stack in pairs as shown in Fig. 4. Each $\pi$-stacked pair (A and B in Fig. 4) has significant overlap of the anthraquinone moiety with

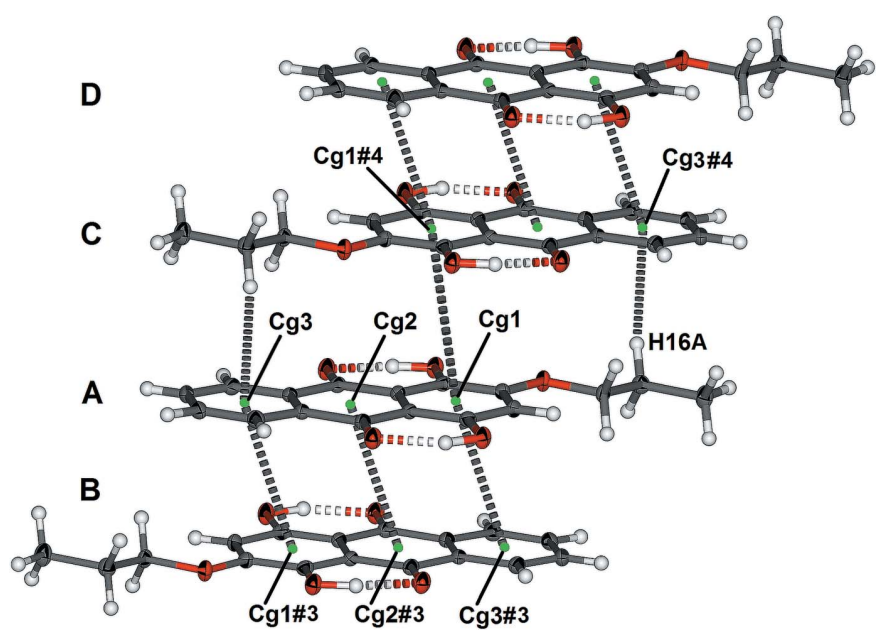

Figure 4

Repetitive $\pi$-stacking of (1). Displacement ellipsoids of non-H atoms are drawn at the $50 \%$ probability level [symmetry codes: (\#1) $-x,-y, 1-z$; (\#2) $2-x, 2-y, 2-z$; (\#3) $1-x, 1-y, 1-z$; (\#4) $2-x, 1-y, 1-z]$.
$C g 1 \cdots C g 3 \# 3, C g 2 \cdots C g 2 \# 3$ [symmetry code: (\#3) $1-x, 1-y$, $1-z ; C g 1, C g 2$ and $C g 3$ are the centroids of the six-membered rings $\mathrm{C} 1-\mathrm{C} 5 / \mathrm{C} 14, \mathrm{C} 5-\mathrm{C} 7 / \mathrm{C} 12-\mathrm{C} 14$ and $\mathrm{C} 7-\mathrm{C} 12$, respectively] distances of 3.607 (1) and 3.569 (1) $⿱$, respectively, with slippages of 1.304 and $1.331 \AA$, respectively. The pairs of $\pi$ stacked molecules of (1) are offset $\pi$-stacked and the alkyl chain has a $\mathrm{C}-\mathrm{H} \cdots \pi$ interaction with one end of the anthraquinone unit, as shown in Fig. 4 (molecules labelled A and C). The C16 ‥Cg3\#4 distance is 3.587 (2) $\AA$ [symmetry code: (\#4) $2-x, 1-y, 1-z]$.

(a)

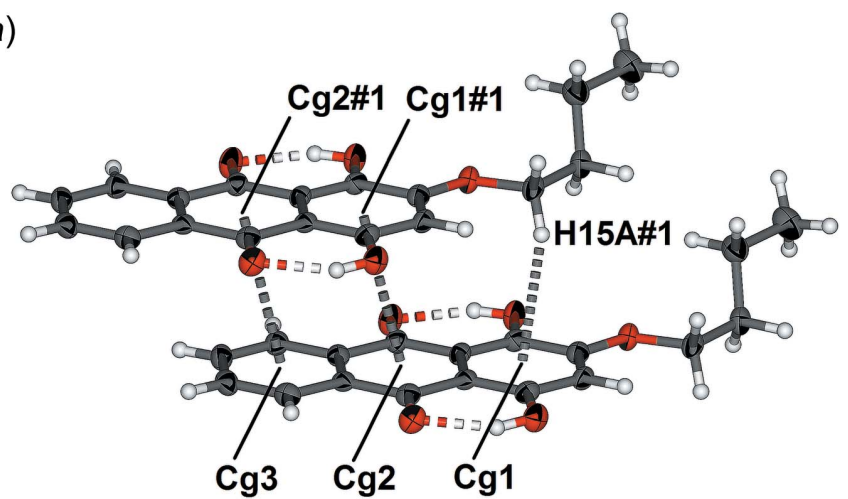

(b)

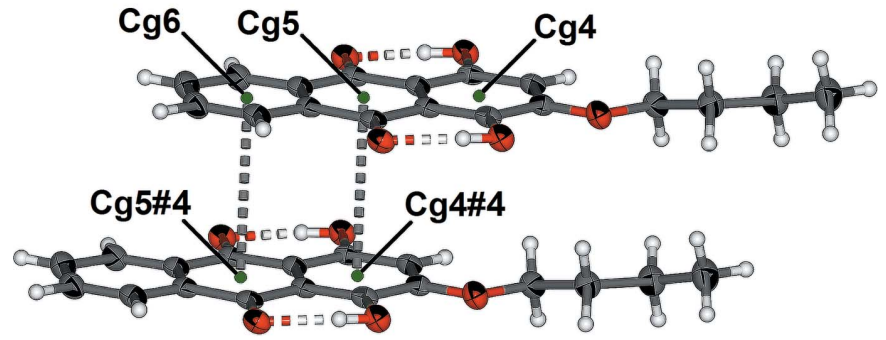

Figure 5

$\pi$-Stacking of the two unique molecules of (2) showing the $\mathrm{C}-\mathrm{H} \cdots \pi$ and $\pi-\pi$ interactions as grey dashed lines. Part $(a)$ shows the $\mathrm{C} 1-\mathrm{C} 14$ anthraquinone unit and $(b)$ the $\mathrm{C} 19-\mathrm{C} 32$ anthraquinone unit. Displacement ellipsoids drawn at the $50 \%$ probability level [symmetry codes: $(\# 1)$ $x-1, y z$; (\#2) $3-x, 1-y, 1-z$; (\#3) $2-x, 1-y, 1-z$; (\#4) $1+x, y, z]$. 
The two unique anthraquinone molecules in the asymmetric unit of (2) offset $\pi$-stack in individual columns. There are three close $\mathrm{C}-\mathrm{H}$. . O contacts (Table 2) between these offset $\pi$-stacked columns. The C...O distances are $3.485(3)$,

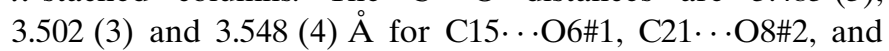
C33...O9\#3. respectively [symmetry codes: (\#1) $x-1, y, z$; (\#2) $3-x, 1-y, 1-z$; (\#3) $2-x, 1-y, 1-z]$. The interactions within each of the two unique sets of $\pi$-stacked molecules are shown in Fig. 5. For the anthraquinone unit defined by $\mathrm{C} 1-\mathrm{C} 14$ (Fig. $5 a$ ), the centroid-to-centroid distances $C g 2 \cdots C g 1 \# 1$ and $C g 3 \cdots C g 2 \# 1$ are 3.521 (2) and 3.517 (2) $\AA$, with slippages of 0.960 and $0.948 \AA$, respectively, where $C g 1, C g 2$ and $C g 3$ are the centroids of the sixmembered rings $\mathrm{C} 1-\mathrm{C} 5 / \mathrm{C} 14, \mathrm{C} 5-\mathrm{C} 7 / \mathrm{C} 12-\mathrm{C} 14$ and $\mathrm{C} 7-\mathrm{C} 12$, respectively. The methylene hydrogen atom $\mathrm{H} 15 A \# 1$ is positioned above centroid $C g 1$ with a $C 15 \cdots C g 1$ distance of 3.690 (3) $\AA$. For the anthraquinone unit defined by C19-C32 (Fig. 5b), the centroid-to-centroid distances $C g 5 \cdots C g 4 \# 4$ and Cg6 ‥Cg5\#4 [symmetry code: (\#4) $1+x, y, z ; C g 4, C g 5$ and Cg6 are the centroids of the C19-C23/C32, C23-C25/C30C32 and C25-C30 rings, respectively] are 3.520 (1) and 4.009 (1) A with slippages of 0.960 and $2.145 \AA$, respectively.

\section{Database survey}

A search of the Cambridge Crystallographic Database (Version 5.38, Nov. 2016; Groom et al., 2016) using Conquest (Bruno et al., 2002) for the anthraquinone ring system with oxygen atoms at positions 1,2 and 4 without restriction on substitution of the other aromatic position, revealed 15 structures. Database entries not including atomic coordinates were excluded. The structure of the parent compound, 1,2,4trihydroxy anthraquinone monohydrate has been reported (refcode QEGNEV; Yatsenko et al., 2000). In addition, structures have been determined for several organic derivatives that were isolated from natural sources. For example, the derivative most closely related to the structures reported here, 1,4-dihydroxy-2-methoxy-7-methylanthracene-9,10-dione, has been isolated from two different fungi and the structure reported [refcodes GEPCOU (She et al., 2006) and GEPCOU01 (Muangsin et al., 2008)]. Complexes of purpurin with rhenium (refcodes CEVNIB, CEVNOH and AVABEF; Sathiyendiran, et al., 2006, 2011), copper [refcode ZOMSEB; Das, et al., 2014), tin (refcodes MOQTAO and MOQTES; de Sousa et al., 2009), calcium and aluminum (refcode LAYBAO; Bergerhoff \& Wunderlich, 1993) have been reported. In each of the reported structures, those compounds with a free hydroxyl group flanking the anthraquinone carbonyl also exhibit the intramolecular hydrogen bond reported for (1) and (2).

\section{Synthesis and crystallization}

Synthesis of 1,4-dihydroxy-2-propoxy anthraquinone (1). In a flask under an atmosphere of argon, a dark red-orange solution of purpurin $(0.26 \mathrm{~g})$ in dimethylformamide $(10 \mathrm{~mL})$ and tetrahydrofuran $(20 \mathrm{~mL})$ was cooled in an ice-salt bath.
Sodium hydride ( $0.081 \mathrm{~g}, 1$ eq.) was added and the resultant violet solution was stirred in the ice bath for 20 minutes. Excess 1-bromopropane (1 mL) was added, a water condenser attached, and the flask was removed from the cooling bath and heated to $353 \mathrm{~K}$ for $24 \mathrm{~h}$. The flask was cooled to room temperature and the solvents evaporated. The crude product was purified by column chromatography with silica gel (0.65$0.40 \mathrm{~mm}$ ) and mixtures of hexane and ethyl acetate of increasing polarity. The eluant was monitored by TLC with a 5:1 mixture of hexane and ethyl acetate. The solvent was evaporated and the product obtained as a red-orange solid (0.15 g). ${ }^{1} \mathrm{H}$ NMR: (400MHz, $\left.\mathrm{CDCl}_{3}\right) \delta 13.56(s, 1 \mathrm{H}), 13.47(s$, $1 \mathrm{H}), 8.33(d d, J=2.0,7.0 \mathrm{~Hz}, 2 \mathrm{H}), 7.84-7.77(m, 2 \mathrm{H}), 6.67(s$, $1 \mathrm{H}), 4.09(t, J=8.0 \mathrm{~Hz}, 2 \mathrm{H}), 1.97(s, J=7.0 \mathrm{~Hz}, 2 \mathrm{H}), 1.11(t, J=$ $7.4 \mathrm{~Hz}, 3 \mathrm{H}) .{ }^{13} \mathrm{C}$ NMR: $189.87,186.98,163.64,159.97,153.30$, $137.17,136.78,136.41,135.95,129.63,129.47,115.07,110.03$, 108.64, 73.84, 24.68, 13.02. Compound (1) crystallized from acetonitrile as large dark-red blocks that included an acetonitrile molecule as a 1:1 solvate. When these blocks were cut to small individual pieces or ground with a mortar and pestle they appeared orange. The crystals lost luster after removal from the mother liquor, presumably due to loss of the acetonitrile.

Synthesis of 4-butoxy-1,2-dihydroxyanthraquinone (2). The same procedure was used with $1 \mathrm{~mL}$ of 1-bromobutane. The compound was isolated as a dark red-purple solid. ${ }^{1} \mathrm{H}$ NMR: $\left(400 \mathrm{MHz}, \mathrm{CDCl}_{3}\right) \delta 13.55(s, 1 \mathrm{H}), 13.46(s, 1 \mathrm{H}), 8.33(d d, J=$ 2.0, $7.0 \mathrm{~Hz}, 2 \mathrm{H}), 7.84-7.76(m, 2 \mathrm{H}), 6.66(s, 1 \mathrm{H}), 4.13(t, J=$ $6.6 \mathrm{~Hz}, 2 \mathrm{H}), 1.92(m, 2 \mathrm{H}), 1.56(m, 2 \mathrm{H}), 1.02(t, J=7.4 \mathrm{~Hz}, 3 \mathrm{H})$. ${ }^{13}$ C NMR: $187.40,184.50,161.22,157.56,150.87,134.72,134.33$, 133.96, 133.51, 107.57, 106.18, 69.73, 30.85, 19.37, 13.98 . Compound (2) was recrystallized from nitromethane as dark red-black blocks. When these blocks were cut to small individual pieces or ground with a mortar and pestle they appeared orange-red.

\section{Refinement}

Crystal data, data collection and structure refinement details are summarized in Table 3. Hydrogen atoms potentially involved in hydrogen-bonding interactions were located by difference methods and initially restrained in the refinement with $\mathrm{O}-\mathrm{H}=0.84(2) \AA$ and with $U_{\text {iso }}(\mathrm{H})=1.2 U_{\text {eq }}(\mathrm{O})$. Other $\mathrm{H}$ atoms were included in the refinement at calculated positions, $\mathrm{C}-\mathrm{H}=0.95 \AA$ for aromatic, $\mathrm{C}-\mathrm{H}=0.99 \AA$ for methylene and $\mathrm{C}-\mathrm{H}=0.98 \AA$ for methyl hydrogens with $U_{\text {iso }}(\mathrm{H})=1.2 U_{\text {eq }}(\mathrm{C})$ for aromatic and methylene $\mathrm{H}$ atoms and $1.5 U_{\text {eq }}(\mathrm{C})$ for methyl $\mathrm{H}$ atoms.

\section{Acknowledgements}

We thank the Missouri State University Provost Incentive Fund that funded the purchase of the X-ray diffractometer.

\section{References}

Barbour, L. J. (2001). J. Supramol. Chem. 1, 189-191.

Bergerhoff, G. \& Wunderlich, C. H. (1993). Z. Kristallogr. 207, 189192. 
Table 3

Experimental details.

(1)

Crystal data

Chemical formula

$M_{\mathrm{r}}$

Crystal system, space group

Temperature $(\mathrm{K})$

$a, b, c(\AA)$

$\alpha, \beta, \gamma\left({ }^{\circ}\right)$

$V\left(\AA^{3}\right)$

Z

Radiation type

$\mu\left(\mathrm{mm}^{-1}\right)$

Crystal size (mm)

Data collection

Diffractometer

Absorption correction

$T_{\min }, T_{\max }$

No. of measured, independent and

observed $[I>2 \sigma(I)]$ reflections

$R_{\text {int }}$

$(\sin \theta / \lambda)_{\max }\left(\AA^{-1}\right)$

Refinement

$R\left[F^{2}>2 \sigma\left(F^{2}\right)\right], w R\left(F^{2}\right), S$

No. of reflections

No. of parameters

No. of restraints

$\mathrm{H}$-atom treatment

$\Delta \rho_{\max }, \Delta \rho_{\min }\left(\mathrm{e} \AA^{-3}\right)$

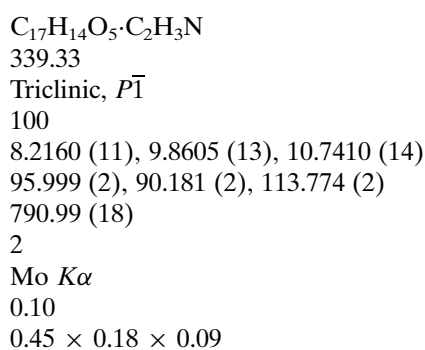

Bruker APEXII CCD

Multi-scan (SADABS; Bruker, 2014)

$0.869,1.000$

10216, 3548, 2718

0.022

0.644

$0.052,0.153,1.06$
3548
234
2
H atoms treated by a mixture of independent
$\quad$ and constrained refinement
$0.71,-0.27$

(2)

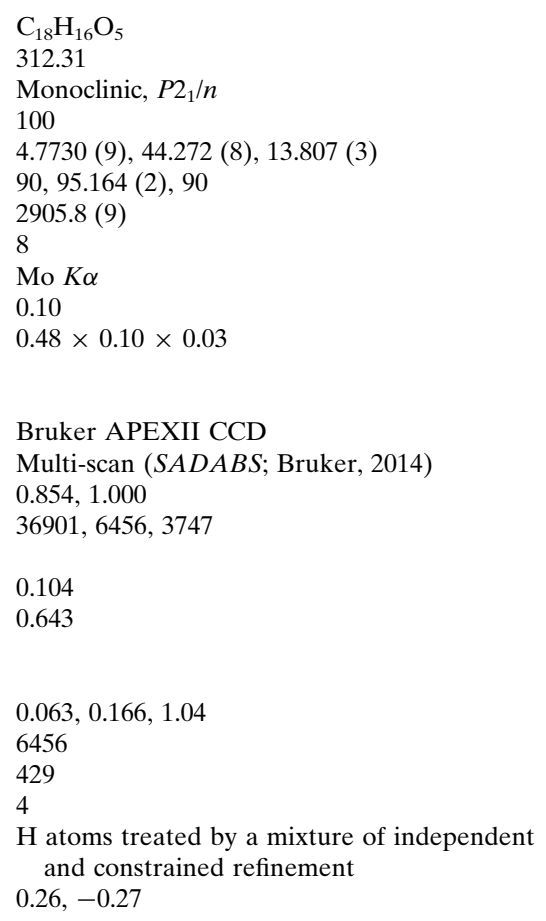

Computer programs: SMART and SAINT (Bruker, 2014), SHELXT2014 (Sheldrick, 2015a), SHELXL2017 (Sheldrick, $2015 b)$ and X-SEED (Barbour, 2001).

Bruker (2014). SMART, SAINT, and SADABS. Bruker AXS Inc., Madison, Wisconsin, USA.

Bruno, I. J., Cole, J. C., Edgington, P. R., Kessler, M., Macrae, C. F., McCabe, P., Pearson, J. \& Taylor, R. (2002). Acta Cryst. B58, 389397.

Das, P., Jain, C. K., Dey, S. K., Saha, R., Chowdhury, A. D., Roychoudhury, S., Kumar, S., Majumder, H. K. \& Das, S. (2014). RSC Adv. 4, 59344-59357.

Groom, C. R., Bruno, I. J., Lightfoot, M. P. \& Ward, S. C. (2016). Acta Cryst. B72, 171-179.

Muangsin, N., Wisetsakdakorn, W., Chaichit, N., Sihanonth, P., Petsom, A. \& Sangvanich, P. (2008). Dyes Pigments, 77, 653-656.

Sathiyendiran, M., Liao, R. T., Thanasekaran, P., Luo, T. T., Venkataramanan, N. S., Lee, G. H., Peng, S. M. \& Lu, K. L. (2006). Inorg. Chem. 45, 10052-10054.
Sathiyendiran, M., Tsai, C. C., Thanasekaran, P., Luo, T. T., Yang, C. I., Lee, G. H., Peng, S. M. \& Lu, K. L. (2011). Chem. Eur. J. 17, 3343-3346.

Schweppe, H. \& Winter, J. (1997). Artists Pigments, 3, edited by E. W. Fitzhugh, pp. 109-142. New York: Oxford University Press.

She, Z.-G., Huang, H.-R., Lin, Y.-C., Vrijmoed, L. L. P. \& Jones, E. B. G. (2006). Acta Cryst. E62, o3737-o3738.

Sheldrick, G. M. (2015a). Acta Cryst. A71, 3-8.

Sheldrick, G. M. (2015b). Acta Cryst. C71, 3-8.

Sousa, A. T. de, Bessler, K. E., Lemos, S. S., Ellena, J. \& Gatto, C. C. (2009). Z. Anorg. Allg. Chem. 635, 106-111.

Yatsenko, A. V., Chernyshev, V. V., Popov, S. I., Sonneveld, E. J. \& Schenk, H. (2000). Dyes Pigments, 45, 169-176. 


\section{supporting information}

Acta Cryst. (2017). E73, 1687-1691 [https://doi.org/10.1107/S2056989017014724]

Synthesis and crystal structures of two purpurin derivatives: 1,4-dihydroxy-2propoxyanthraquinone and 2-butoxy-1,4-dihydroxyanthraquinone

\section{Eric Bosch and Emily N. McClain}

\section{Computing details}

For both structures, data collection: SMART (Bruker, 2014); cell refinement: SMART (Bruker, 2014); data reduction: SAINT (Bruker, 2014); program(s) used to solve structure: SHELXT2014 (Sheldrick, 2015a); program(s) used to refine structure: SHELXL2017 (Sheldrick, 2015b); molecular graphics: X-SEED (Barbour, 2001); software used to prepare material for publication: X-SEED (Barbour, 2001).

1,4-Dihydroxy-2-propoxyanthraquinone acetonitrile monosolvate (1)

\section{Crystal data}

$\mathrm{C}_{17} \mathrm{H}_{14} \mathrm{O}_{5} \cdot \mathrm{C}_{2} \mathrm{H}_{3} \mathrm{~N}$

$M_{r}=339.33$

Triclinic, $P \overline{1}$

$a=8.2160$ (11) $\AA$

$b=9.8605(13) \AA$

$c=10.7410(14) \AA$

$\alpha=95.999(2)^{\circ}$

$\beta=90.181(2)^{\circ}$

$\gamma=113.774(2)^{\circ}$

$V=790.99(18) \AA^{3}$

\section{Data collection}

Bruker APEXII CCD diffractometer

Radiation source: fine-focus sealed tube Graphite monochromator

Detector resolution: 8.3660 pixels $\mathrm{mm}^{-1}$

phi and $\omega$ scans

Absorption correction: multi-scan

(SADABS; Bruker, 2014)

$T_{\min }=0.869, T_{\max }=1.000$

\section{Refinement}

Refinement on $F^{2}$

Least-squares matrix: full

$R\left[F^{2}>2 \sigma\left(F^{2}\right)\right]=0.052$

$w R\left(F^{2}\right)=0.153$

$S=1.06$

3548 reflections

234 parameters

2 restraints

$$
\begin{aligned}
& Z=2 \\
& F(000)=356 \\
& D_{\mathrm{x}}=1.425 \mathrm{Mg} \mathrm{m}^{-3} \\
& \text { Mo } K \alpha \text { radiation, } \lambda=0.71073 \AA \\
& \text { Cell parameters from } 3016 \text { reflect } \\
& \theta=2.7-27.2^{\circ} \\
& \mu=0.10 \mathrm{~mm}^{-1} \\
& T=100 \mathrm{~K} \\
& \text { Cut irregular block, orange } \\
& 0.45 \times 0.18 \times 0.09 \mathrm{~mm}
\end{aligned}
$$

Cell parameters from 3016 reflections

10216 measured reflections 3548 independent reflections 2718 reflections with $I>2 \sigma(I)$

$R_{\text {int }}=0.022$

$\theta_{\max }=27.3^{\circ}, \theta_{\min }=1.9^{\circ}$

$h=-10 \rightarrow 10$

$k=-12 \rightarrow 12$

$l=-13 \rightarrow 13$

Hydrogen site location: mixed

$\mathrm{H}$ atoms treated by a mixture of independent and constrained refinement

$w=1 /\left[\sigma^{2}\left(F_{\mathrm{o}}^{2}\right)+(0.0962 P)^{2}+0.0684 P\right]$

where $P=\left(F_{\mathrm{o}}^{2}+2 F_{\mathrm{c}}^{2}\right) / 3$

$(\Delta / \sigma)_{\max }<0.001$

$\Delta \rho_{\max }=0.71$ e $\AA^{-3}$

$\Delta \rho_{\min }=-0.27$ e $\AA^{-3}$ 


\section{Special details}

Geometry. All esds (except the esd in the dihedral angle between two 1.s. planes) are estimated using the full covariance matrix. The cell esds are taken into account individually in the estimation of esds in distances, angles and torsion angles; correlations between esds in cell parameters are only used when they are defined by crystal symmetry. An approximate (isotropic) treatment of cell esds is used for estimating esds involving l.s. planes.

Fractional atomic coordinates and isotropic or equivalent isotropic displacement parameters $\left(\AA^{2}\right)$

\begin{tabular}{|c|c|c|c|c|}
\hline & $x$ & $y$ & $z$ & $U_{\text {iso }} * / U_{\text {eq }}$ \\
\hline $\mathrm{O} 1$ & 0.93351 (13) & $0.54640(11)$ & $0.31245(9)$ & $0.0224(2)$ \\
\hline $\mathrm{H} 1 \mathrm{O}$ & $0.943(2)$ & $0.6376(15)$ & $0.3329(15)$ & $0.027 *$ \\
\hline N1 & $0.69308(19)$ & $1.25060(16)$ & 0.93564 (13) & $0.0376(4)$ \\
\hline $\mathrm{C} 1$ & $0.83016(17)$ & $0.46925(15)$ & $0.39909(12)$ & $0.0179(3)$ \\
\hline $\mathrm{O} 2$ & $0.85708(12)$ & $0.26110(10)$ & $0.29025(9)$ & $0.0216(2)$ \\
\hline $\mathrm{C} 2$ & $0.78580(17)$ & $0.31187(15)$ & $0.38774(12)$ & $0.0186(3)$ \\
\hline $\mathrm{O} 3$ & $0.50110(13)$ & $0.19718(10)$ & $0.64607(9)$ & $0.0231(3)$ \\
\hline $\mathrm{H} 3 \mathrm{O}$ & $0.471(2)$ & $0.2555(17)$ & $0.6993(14)$ & $0.028 *$ \\
\hline $\mathrm{C} 3$ & $0.67840(17)$ & $0.22550(15)$ & $0.47188(12)$ & $0.0190(3)$ \\
\hline H3 & 0.650425 & 0.121543 & 0.464073 & $0.023 *$ \\
\hline $\mathrm{O} 4$ & $0.48044(13)$ & 0.42907 (11) & $0.76272(9)$ & $0.0236(2)$ \\
\hline $\mathrm{C} 4$ & 0.60915 (17) & $0.28978(15)$ & $0.56995(12)$ & $0.0184(3)$ \\
\hline O5 & $0.90743(13)$ & $0.77360(10)$ & $0.43289(9)$ & $0.0237(3)$ \\
\hline C5 & $0.65148(17)$ & $0.44303(14)$ & $0.58446(12)$ & 0.0168 (3) \\
\hline C6 & $0.58069(17)$ & $0.50732(15)$ & $0.68671(12)$ & $0.0186(3)$ \\
\hline $\mathrm{C} 7$ & 0.63155 (17) & $0.67078(15)$ & $0.70039(12)$ & $0.0182(3)$ \\
\hline $\mathrm{C} 8$ & $0.57026(18)$ & $0.73660(16)$ & 0.80017 (12) & 0.0215 (3) \\
\hline H8 & 0.496307 & 0.676508 & 0.858499 & $0.026^{*}$ \\
\hline C9 & $0.61668(18)$ & $0.88946(16)$ & $0.81480(13)$ & $0.0230(3)$ \\
\hline H9 & 0.577257 & 0.934122 & 0.884204 & $0.028 *$ \\
\hline $\mathrm{C} 10$ & $0.72114(18)$ & $0.97713(15)$ & $0.72752(13)$ & $0.0232(3)$ \\
\hline H10 & 0.750414 & 1.081320 & 0.736488 & $0.028 *$ \\
\hline $\mathrm{C} 11$ & $0.78274(18)$ & $0.91324(15)$ & $0.62752(13)$ & $0.0211(3)$ \\
\hline H11 & 0.854261 & 0.973685 & 0.568420 & $0.025 *$ \\
\hline $\mathrm{C} 12$ & $0.73939(17)$ & $0.75953(14)$ & $0.61376(12)$ & $0.0175(3)$ \\
\hline $\mathrm{C} 13$ & 0.80994 (17) & $0.69324(15)$ & $0.50858(12)$ & $0.0185(3)$ \\
\hline $\mathrm{C} 14$ & $0.76399(17)$ & $0.53320(14)$ & $0.49710(12)$ & $0.0171(3)$ \\
\hline $\mathrm{C} 15$ & $0.80376(18)$ & $0.10079(14)$ & $0.26995(12)$ & 0.0197 (3) \\
\hline H15A & 0.672284 & 0.048972 & 0.262662 & $0.024 *$ \\
\hline H15B & 0.847744 & 0.066928 & 0.341400 & $0.024^{*}$ \\
\hline C16 & $0.88231(18)$ & $0.06548(15)$ & $0.15066(12)$ & $0.0216(3)$ \\
\hline $\mathrm{H} 16 \mathrm{~A}$ & 1.013724 & 0.117694 & 0.158502 & $0.026^{*}$ \\
\hline H16B & 0.839009 & 0.100713 & 0.079785 & $0.026^{*}$ \\
\hline $\mathrm{C} 17$ & 0.8285 (2) & $-0.10258(15)$ & $0.12525(13)$ & $0.0259(3)$ \\
\hline H17A & 0.864454 & -0.138135 & 0.198037 & $0.039 *$ \\
\hline H17B & 0.887518 & -0.124293 & 0.051405 & $0.039 *$ \\
\hline $\mathrm{H} 17 \mathrm{C}$ & 0.699127 & -0.153202 & 0.110002 & $0.039 *$ \\
\hline $\mathrm{C} 18$ & 0.7439 (2) & $1.37684(18)$ & $0.95263(14)$ & $0.0300(3)$ \\
\hline C19 & 0.8085 (2) & $1.53842(19)$ & $0.97699(19)$ & $0.0451(5)$ \\
\hline
\end{tabular}


supporting information

\begin{tabular}{lllll} 
H19A & 0.707605 & 1.567300 & 0.974361 & $0.068^{*}$ \\
H19B & 0.891236 & 1.584642 & 0.913041 & $0.068^{*}$ \\
H19C & 0.870055 & 1.572114 & 1.059963 & $0.068^{*}$ \\
\hline
\end{tabular}

Atomic displacement parameters $\left(\AA^{2}\right)$

\begin{tabular}{lllllll}
\hline & $U^{11}$ & $U^{22}$ & $U^{33}$ & $U^{12}$ & $U^{13}$ & $U^{23}$ \\
\hline O1 & $0.0257(5)$ & $0.0171(5)$ & $0.0230(5)$ & $0.0076(4)$ & $0.0075(4)$ & $0.0008(4)$ \\
N1 & $0.0451(8)$ & $0.0342(8)$ & $0.0356(8)$ & $0.0199(7)$ & $0.0016(6)$ & $-0.0023(6)$ \\
C1 & $0.0159(6)$ & $0.0174(6)$ & $0.0186(6)$ & $0.0052(5)$ & $0.0005(5)$ & $0.0013(5)$ \\
O2 & $0.0243(5)$ & $0.0157(5)$ & $0.0244(5)$ & $0.0086(4)$ & $0.0078(4)$ & $-0.0012(4)$ \\
C2 & $0.0173(6)$ & $0.0200(7)$ & $0.0190(6)$ & $0.0092(5)$ & $0.0005(5)$ & $-0.0026(5)$ \\
O3 & $0.0278(5)$ & $0.0164(5)$ & $0.0249(5)$ & $0.0086(4)$ & $0.0091(4)$ & $0.0029(4)$ \\
C3 & $0.0188(6)$ & $0.0151(6)$ & $0.0230(7)$ & $0.0071(5)$ & $0.0002(5)$ & $0.0003(5)$ \\
O4 & $0.0263(5)$ & $0.0202(5)$ & $0.0239(5)$ & $0.0092(4)$ & $0.0069(4)$ & $0.0018(4)$ \\
C4 & $0.0176(6)$ & $0.0184(6)$ & $0.0194(6)$ & $0.0076(5)$ & $0.0011(5)$ & $0.0021(5)$ \\
O5 & $0.0271(5)$ & $0.0170(5)$ & $0.0247(5)$ & $0.0066(4)$ & $0.0067(4)$ & $0.0018(4)$ \\
C5 & $0.0165(6)$ & $0.0167(6)$ & $0.0170(6)$ & $0.0071(5)$ & $-0.0007(5)$ & $0.0000(5)$ \\
C6 & $0.0178(6)$ & $0.0193(7)$ & $0.0193(6)$ & $0.0086(5)$ & $0.0004(5)$ & $0.0007(5)$ \\
C7 & $0.0168(6)$ & $0.0182(7)$ & $0.0195(6)$ & $0.0078(5)$ & $-0.0019(5)$ & $-0.0012(5)$ \\
C8 & $0.0225(7)$ & $0.0233(7)$ & $0.0192(6)$ & $0.0106(6)$ & $0.0007(5)$ & $-0.0004(5)$ \\
C9 & $0.0228(7)$ & $0.0245(7)$ & $0.0230(7)$ & $0.0131(6)$ & $-0.0025(5)$ & $-0.0056(5)$ \\
C10 & $0.0235(7)$ & $0.0183(7)$ & $0.0282(7)$ & $0.0107(6)$ & $-0.0037(6)$ & $-0.0042(5)$ \\
C11 & $0.0207(7)$ & $0.0189(6)$ & $0.0236(7)$ & $0.0084(5)$ & $-0.0014(5)$ & $0.0008(5)$ \\
C12 & $0.0167(6)$ & $0.0173(7)$ & $0.0180(6)$ & $0.0073(5)$ & $-0.0029(5)$ & $-0.0018(5)$ \\
C13 & $0.0175(6)$ & $0.0170(7)$ & $0.0201(6)$ & $0.0065(5)$ & $-0.0009(5)$ & $0.0006(5)$ \\
C14 & $0.0161(6)$ & $0.0156(7)$ & $0.0185(6)$ & $0.0061(5)$ & $-0.0015(5)$ & $-0.0012(5)$ \\
C15 & $0.0206(7)$ & $0.0139(6)$ & $0.0233(7)$ & $0.0065(5)$ & $0.0030(5)$ & $-0.0017(5)$ \\
C16 & $0.0223(7)$ & $0.0212(7)$ & $0.0208(7)$ & $0.0089(5)$ & $0.0021(5)$ & $-0.0006(5)$ \\
C17 & $0.0319(8)$ & $0.0222(7)$ & $0.0228(7)$ & $0.0113(6)$ & $0.0068(6)$ & $-0.0028(5)$ \\
C18 & $0.0311(8)$ & $0.0349(9)$ & $0.0271(7)$ & $0.0178(7)$ & $-0.0004(6)$ & $-0.0015(6)$ \\
C19 & $0.0445(10)$ & $0.0300(9)$ & $0.0596(11)$ & $0.0163(8)$ & $-0.0157(9)$ & $-0.0045(8)$ \\
& & & & & & \\
& & & & &
\end{tabular}

Geometric parameters $\left(\AA,{ }^{\circ}\right)$

\begin{tabular}{llll}
\hline $\mathrm{O} 1-\mathrm{C} 1$ & $1.3410(15)$ & $\mathrm{C} 9-\mathrm{C} 10$ & $1.392(2)$ \\
$\mathrm{O} 1-\mathrm{H} 1 \mathrm{O}$ & $0.875(13)$ & $\mathrm{C} 9-\mathrm{H} 9$ & 0.9500 \\
$\mathrm{~N} 1-\mathrm{C} 18$ & $1.136(2)$ & $\mathrm{C} 10-\mathrm{C} 11$ & $1.3877(19)$ \\
$\mathrm{C} 1-\mathrm{C} 14$ & $1.3938(19)$ & $\mathrm{C} 10-\mathrm{H} 10$ & 0.9500 \\
$\mathrm{C} 1-\mathrm{C} 2$ & $1.4353(19)$ & $\mathrm{C} 11-\mathrm{C} 12$ & $1.4013(18)$ \\
$\mathrm{O} 2-\mathrm{C} 2$ & $1.3493(16)$ & $\mathrm{C} 11-\mathrm{H} 11$ & 0.9500 \\
$\mathrm{O} 2-\mathrm{C} 15$ & $1.4516(15)$ & $\mathrm{C} 12-\mathrm{C} 13$ & $1.4814(19)$ \\
$\mathrm{C} 2-\mathrm{C} 3$ & $1.3714(19)$ & $\mathrm{C} 13-\mathrm{C} 14$ & $1.4584(19)$ \\
$\mathrm{O} 3-\mathrm{C} 4$ & $1.3407(15)$ & $\mathrm{C} 15-\mathrm{C} 16$ & $1.5075(18)$ \\
$\mathrm{O} 3-\mathrm{H} 3 \mathrm{O}$ & $0.874(14)$ & $\mathrm{C} 15-\mathrm{H} 15 \mathrm{~A}$ & 0.9900 \\
$\mathrm{C} 3-\mathrm{C} 4$ & $1.4106(18)$ & $\mathrm{C} 15-\mathrm{H} 15 \mathrm{~B}$ & 0.9900 \\
$\mathrm{C} 3-\mathrm{H} 3$ & 0.9500 & $\mathrm{C} 16-\mathrm{C} 17$ & $1.5265(18)$ \\
$\mathrm{O} 4-\mathrm{C} 6$ & $1.2505(16)$ & $\mathrm{C} 16-\mathrm{H} 16 \mathrm{~A}$ & 0.9900
\end{tabular}




\begin{tabular}{|c|c|c|c|}
\hline $\mathrm{C} 4-\mathrm{C} 5$ & $1.3983(18)$ & $\mathrm{C} 16-\mathrm{H} 16 \mathrm{~B}$ & 0.9900 \\
\hline $\mathrm{O} 5-\mathrm{C} 13$ & $1.2480(16)$ & C17-H17A & 0.9800 \\
\hline $\mathrm{C} 5-\mathrm{C} 14$ & $1.4292(19)$ & $\mathrm{C} 17-\mathrm{H} 17 \mathrm{~B}$ & 0.9800 \\
\hline $\mathrm{C} 5-\mathrm{C} 6$ & $1.4498(18)$ & $\mathrm{C} 17-\mathrm{H} 17 \mathrm{C}$ & 0.9800 \\
\hline $\mathrm{C} 6-\mathrm{C} 7$ & $1.4836(18)$ & $\mathrm{C} 18-\mathrm{C} 19$ & $1.456(2)$ \\
\hline $\mathrm{C} 7-\mathrm{C} 8$ & $1.3947(18)$ & C19-H19A & 0.9800 \\
\hline $\mathrm{C} 7-\mathrm{C} 12$ & $1.4025(18)$ & C19-H19B & 0.9800 \\
\hline $\mathrm{C} 8-\mathrm{C} 9$ & $1.3887(19)$ & $\mathrm{C} 19-\mathrm{H} 19 \mathrm{C}$ & 0.9800 \\
\hline $\mathrm{C} 8-\mathrm{H} 8$ & 0.9500 & & \\
\hline $\mathrm{C} 1-\mathrm{O} 1-\mathrm{H} 1 \mathrm{O}$ & $104.0(11)$ & $\mathrm{C} 12-\mathrm{C} 11-\mathrm{H} 11$ & 120.0 \\
\hline $\mathrm{O} 1-\mathrm{C} 1-\mathrm{C} 14$ & $123.72(12)$ & $\mathrm{C} 11-\mathrm{C} 12-\mathrm{C} 7$ & $119.64(12)$ \\
\hline $\mathrm{O} 1-\mathrm{C} 1-\mathrm{C} 2$ & $117.06(11)$ & $\mathrm{C} 11-\mathrm{C} 12-\mathrm{C} 13$ & $119.48(12)$ \\
\hline $\mathrm{C} 14-\mathrm{C} 1-\mathrm{C} 2$ & $119.22(12)$ & $\mathrm{C} 7-\mathrm{C} 12-\mathrm{C} 13$ & $120.88(12)$ \\
\hline $\mathrm{C} 2-\mathrm{O} 2-\mathrm{C} 15$ & $116.12(10)$ & $\mathrm{O} 5-\mathrm{C} 13-\mathrm{C} 14$ & $121.35(12)$ \\
\hline $\mathrm{O} 2-\mathrm{C} 2-\mathrm{C} 3$ & $125.11(12)$ & $\mathrm{O} 5-\mathrm{C} 13-\mathrm{C} 12$ & $120.31(12)$ \\
\hline $\mathrm{O} 2-\mathrm{C} 2-\mathrm{C} 1$ & $114.62(12)$ & $\mathrm{C} 14-\mathrm{C} 13-\mathrm{C} 12$ & $118.33(12)$ \\
\hline $\mathrm{C} 3-\mathrm{C} 2-\mathrm{C} 1$ & $120.27(12)$ & $\mathrm{C} 1-\mathrm{C} 14-\mathrm{C} 5$ & $120.48(12)$ \\
\hline $\mathrm{C} 4-\mathrm{O} 3-\mathrm{H} 3 \mathrm{O}$ & $104.0(11)$ & $\mathrm{C} 1-\mathrm{C} 14-\mathrm{C} 13$ & $119.04(12)$ \\
\hline $\mathrm{C} 2-\mathrm{C} 3-\mathrm{C} 4$ & $120.53(12)$ & $\mathrm{C} 5-\mathrm{C} 14-\mathrm{C} 13$ & $120.47(12)$ \\
\hline $\mathrm{C} 2-\mathrm{C} 3-\mathrm{H} 3$ & 119.7 & $\mathrm{O} 2-\mathrm{C} 15-\mathrm{C} 16$ & $107.99(10)$ \\
\hline $\mathrm{C} 4-\mathrm{C} 3-\mathrm{H} 3$ & 119.7 & $\mathrm{O} 2-\mathrm{C} 15-\mathrm{H} 15 \mathrm{~A}$ & 110.1 \\
\hline $\mathrm{O} 3-\mathrm{C} 4-\mathrm{C} 5$ & $122.48(11)$ & $\mathrm{C} 16-\mathrm{C} 15-\mathrm{H} 15 \mathrm{~A}$ & 110.1 \\
\hline $\mathrm{O} 3-\mathrm{C} 4-\mathrm{C} 3$ & $116.90(12)$ & $\mathrm{O} 2-\mathrm{C} 15-\mathrm{H} 15 \mathrm{~B}$ & 110.1 \\
\hline $\mathrm{C} 5-\mathrm{C} 4-\mathrm{C} 3$ & $120.61(12)$ & $\mathrm{C} 16-\mathrm{C} 15-\mathrm{H} 15 \mathrm{~B}$ & 110.1 \\
\hline $\mathrm{C} 4-\mathrm{C} 5-\mathrm{C} 14$ & $118.88(12)$ & $\mathrm{H} 15 \mathrm{~A}-\mathrm{C} 15-\mathrm{H} 15 \mathrm{~B}$ & 108.4 \\
\hline $\mathrm{C} 4-\mathrm{C} 5-\mathrm{C} 6$ & $119.80(12)$ & $\mathrm{C} 15-\mathrm{C} 16-\mathrm{C} 17$ & $109.75(11)$ \\
\hline $\mathrm{C} 14-\mathrm{C} 5-\mathrm{C} 6$ & $121.32(12)$ & $\mathrm{C} 15-\mathrm{C} 16-\mathrm{H} 16 \mathrm{~A}$ & 109.7 \\
\hline $\mathrm{O} 4-\mathrm{C} 6-\mathrm{C} 5$ & $121.78(12)$ & $\mathrm{C} 17-\mathrm{C} 16-\mathrm{H} 16 \mathrm{~A}$ & 109.7 \\
\hline $\mathrm{O} 4-\mathrm{C} 6-\mathrm{C} 7$ & $120.05(11)$ & $\mathrm{C} 15-\mathrm{C} 16-\mathrm{H} 16 \mathrm{~B}$ & 109.7 \\
\hline $\mathrm{C} 5-\mathrm{C} 6-\mathrm{C} 7$ & $118.17(12)$ & $\mathrm{C} 17-\mathrm{C} 16-\mathrm{H} 16 \mathrm{~B}$ & 109.7 \\
\hline $\mathrm{C} 8-\mathrm{C} 7-\mathrm{C} 12$ & $119.70(12)$ & $\mathrm{H} 16 \mathrm{~A}-\mathrm{C} 16-\mathrm{H} 16 \mathrm{~B}$ & 108.2 \\
\hline $\mathrm{C} 8-\mathrm{C} 7-\mathrm{C} 6$ & $119.52(12)$ & $\mathrm{C} 16-\mathrm{C} 17-\mathrm{H} 17 \mathrm{~A}$ & 109.5 \\
\hline $\mathrm{C} 12-\mathrm{C} 7-\mathrm{C} 6$ & $120.79(12)$ & $\mathrm{C} 16-\mathrm{C} 17-\mathrm{H} 17 \mathrm{~B}$ & 109.5 \\
\hline $\mathrm{C} 9-\mathrm{C} 8-\mathrm{C} 7$ & $120.41(13)$ & $\mathrm{H} 17 \mathrm{~A}-\mathrm{C} 17-\mathrm{H} 17 \mathrm{~B}$ & 109.5 \\
\hline $\mathrm{C} 9-\mathrm{C} 8-\mathrm{H} 8$ & 119.8 & $\mathrm{C} 16-\mathrm{C} 17-\mathrm{H} 17 \mathrm{C}$ & 109.5 \\
\hline $\mathrm{C} 7-\mathrm{C} 8-\mathrm{H} 8$ & 119.8 & $\mathrm{H} 17 \mathrm{~A}-\mathrm{C} 17-\mathrm{H} 17 \mathrm{C}$ & 109.5 \\
\hline $\mathrm{C} 8-\mathrm{C} 9-\mathrm{C} 10$ & $119.86(12)$ & $\mathrm{H} 17 \mathrm{~B}-\mathrm{C} 17-\mathrm{H} 17 \mathrm{C}$ & 109.5 \\
\hline $\mathrm{C} 8-\mathrm{C} 9-\mathrm{H} 9$ & 120.1 & $\mathrm{~N} 1-\mathrm{C} 18-\mathrm{C} 19$ & $178.89(17)$ \\
\hline $\mathrm{C} 10-\mathrm{C} 9-\mathrm{H} 9$ & 120.1 & $\mathrm{C} 18-\mathrm{C} 19-\mathrm{H} 19 \mathrm{~A}$ & 109.5 \\
\hline $\mathrm{C} 11-\mathrm{C} 10-\mathrm{C} 9$ & $120.40(12)$ & $\mathrm{C} 18-\mathrm{C} 19-\mathrm{H} 19 \mathrm{~B}$ & 109.5 \\
\hline $\mathrm{C} 11-\mathrm{C} 10-\mathrm{H} 10$ & 119.8 & $\mathrm{H} 19 \mathrm{~A}-\mathrm{C} 19-\mathrm{H} 19 \mathrm{~B}$ & 109.5 \\
\hline $\mathrm{C} 9-\mathrm{C} 10-\mathrm{H} 10$ & 119.8 & $\mathrm{C} 18-\mathrm{C} 19-\mathrm{H} 19 \mathrm{C}$ & 109.5 \\
\hline $\mathrm{C} 10-\mathrm{C} 11-\mathrm{C} 12$ & $119.97(13)$ & $\mathrm{H} 19 \mathrm{~A}-\mathrm{C} 19-\mathrm{H} 19 \mathrm{C}$ & 109.5 \\
\hline $\mathrm{C} 10-\mathrm{C} 11-\mathrm{H} 11$ & 120.0 & $\mathrm{H} 19 \mathrm{~B}-\mathrm{C} 19-\mathrm{H} 19 \mathrm{C}$ & 109.5 \\
\hline $\mathrm{C} 15-\mathrm{O} 2-\mathrm{C} 2-\mathrm{C} 3$ & $4.50(19)$ & $\mathrm{C} 9-\mathrm{C} 10-\mathrm{C} 11-\mathrm{C} 12$ & $0.2(2)$ \\
\hline $\mathrm{C} 15-\mathrm{O} 2-\mathrm{C} 2-\mathrm{C} 1$ & $-175.30(10)$ & $\mathrm{C} 10-\mathrm{C} 11-\mathrm{C} 12-\mathrm{C} 7$ & $0.9(2)$ \\
\hline
\end{tabular}




$\begin{array}{llll}\mathrm{O} 1-\mathrm{C} 1-\mathrm{C} 2-\mathrm{O} 2 & 0.80(18) & \mathrm{C} 10-\mathrm{C} 11-\mathrm{C} 12-\mathrm{C} 13 & -178.36(11) \\ \mathrm{C} 14-\mathrm{C} 1-\mathrm{C} 2-\mathrm{O} 2 & -179.69(11) & \mathrm{C} 8-\mathrm{C} 7-\mathrm{C} 12-\mathrm{C} 11 & -0.8(2) \\ \mathrm{O} 1-\mathrm{C} 1-\mathrm{C} 2-\mathrm{C} 3 & -179.01(11) & \mathrm{C} 6-\mathrm{C} 7-\mathrm{C} 12-\mathrm{C} 11 & 178.81(11) \\ \mathrm{C} 14-\mathrm{C} 1-\mathrm{C} 2-\mathrm{C} 3 & 0.5(2) & \mathrm{C} 8-\mathrm{C} 7-\mathrm{C} 12-\mathrm{C} 13 & 178.54(11) \\ \mathrm{O} 2-\mathrm{C} 2-\mathrm{C} 3-\mathrm{C} 4 & -179.31(12) & \mathrm{C} 6-\mathrm{C} 7-\mathrm{C} 12-\mathrm{C} 13 & -1.9(2) \\ \mathrm{C} 1-\mathrm{C} 2-\mathrm{C} 3-\mathrm{C} 4 & 0.5(2) & \mathrm{C} 11-\mathrm{C} 12-\mathrm{C} 13-\mathrm{O} 5 & 0.1(2) \\ \mathrm{C} 2-\mathrm{C} 3-\mathrm{C} 4-\mathrm{O} 3 & 178.11(11) & \mathrm{C} 7-\mathrm{C} 12-\mathrm{C} 13-\mathrm{O} 5 & -179.19(11) \\ \mathrm{C} 2-\mathrm{C} 3-\mathrm{C} 4-\mathrm{C} 5 & -1.2(2) & \mathrm{C} 11-\mathrm{C} 12-\mathrm{C} 13-\mathrm{C} 14 & 179.48(11) \\ \mathrm{O} 3-\mathrm{C} 4-\mathrm{C} 5-\mathrm{C} 14 & -178.34(11) & \mathrm{C} 7-\mathrm{C} 12-\mathrm{C} 13-\mathrm{C} 14 & 0.19(19) \\ \mathrm{C} 3-\mathrm{C} 4-\mathrm{C} 5-\mathrm{C} 14 & 0.9(2) & \mathrm{O} 1-\mathrm{C} 1-\mathrm{C} 14-\mathrm{C} 5 & 178.71(11) \\ \mathrm{O} 3-\mathrm{C} 4-\mathrm{C} 5-\mathrm{C} 6 & 1.4(2) & \mathrm{C} 2-\mathrm{C} 1-\mathrm{C} 14-\mathrm{C} 5 & -0.8(2) \\ \mathrm{C} 3-\mathrm{C} 4-\mathrm{C} 5-\mathrm{C} 6 & -179.28(11) & \mathrm{O} 1-\mathrm{C} 1-\mathrm{C} 14-\mathrm{C} 13 & -0.8(2) \\ \mathrm{C} 4-\mathrm{C} 5-\mathrm{C} 6-\mathrm{O} 4 & -0.6(2) & \mathrm{C} 2-\mathrm{C} 1-\mathrm{C} 14-\mathrm{C} 13 & 179.77(11) \\ \mathrm{C} 14-\mathrm{C} 5-\mathrm{C} 6-\mathrm{O} 4 & 179.13(11) & \mathrm{C} 4-\mathrm{C} 5-\mathrm{C} 14-\mathrm{C} 1 & 0.1(2) \\ \mathrm{C} 4-\mathrm{C} 5-\mathrm{C} 6-\mathrm{C} 7 & 178.82(11) & \mathrm{C} 6-\mathrm{C} 5-\mathrm{C} 14-\mathrm{C} 1 & -179.73(11) \\ \mathrm{C} 14-\mathrm{C} 5-\mathrm{C} 6-\mathrm{C} 7 & -1.4(2) & \mathrm{C} 4-\mathrm{C} 5-\mathrm{C} 14-\mathrm{C} 13 & 179.51(11) \\ \mathrm{O} 4-\mathrm{C} 6-\mathrm{C} 7-\mathrm{C} 8 & 1.5(2) & \mathrm{C} 6-\mathrm{C} 5-\mathrm{C} 14-\mathrm{C} 13 & -0.3(2) \\ \mathrm{C} 5-\mathrm{C} 6-\mathrm{C} 7-\mathrm{C} 8 & -177.94(11) & \mathrm{O} 5-\mathrm{C} 13-\mathrm{C} 14-\mathrm{C} 1 & -0.3(2) \\ \mathrm{O} 4-\mathrm{C} 6-\mathrm{C} 7-\mathrm{C} 12 & -178.03(11) & \mathrm{C} 12-\mathrm{C} 13-\mathrm{C} 14-\mathrm{C} 1 & -179.62(11) \\ \mathrm{C} 5-\mathrm{C} 6-\mathrm{C} 7-\mathrm{C} 12 & 2.49(19) & \mathrm{O} 5-\mathrm{C} 13-\mathrm{C} 14-\mathrm{C} 5 & -179.72(11) \\ \mathrm{C} 12-\mathrm{C} 7-\mathrm{C} 8-\mathrm{C} 9 & -0.6(2) & \mathrm{C} 12-\mathrm{C} 13-\mathrm{C} 14-\mathrm{C} 5 & 0.91(19) \\ \mathrm{C} 6-\mathrm{C} 7-\mathrm{C} 8-\mathrm{C} 9 & 179.85(11) & \mathrm{C} 2-\mathrm{O} 2-\mathrm{C} 15-\mathrm{C} 16 & 174.51(10) \\ \mathrm{C} 7-\mathrm{C} 8-\mathrm{C} 9-\mathrm{C} 10 & 1.7(2) & \mathrm{O} 2-\mathrm{C} 15-\mathrm{C} 16-\mathrm{C} 17 & -179.69(10) \\ \mathrm{C} 8-\mathrm{C} 9-\mathrm{C} 10-\mathrm{C} 11 & -1.5(2) & & \end{array}$

Hydrogen-bond geometry $\left(\AA,{ }^{\circ}\right)$

\begin{tabular}{lllll}
\hline$D-\mathrm{H} \cdots A$ & $D-\mathrm{H}$ & $\mathrm{H} \cdots A$ & $D \cdots A$ & $D-\mathrm{H} \cdots A$ \\
\hline $\mathrm{O} 1-\mathrm{H} 1 O \cdots \mathrm{O} 5$ & $0.88(1)$ & $1.75(1)$ & $2.5537(13)$ & $152(2)$ \\
$\mathrm{O} 3-\mathrm{H} 3 O \cdots \mathrm{O} 4$ & $0.87(1)$ & $1.75(1)$ & $2.5578(13)$ & $153(2)$ \\
$\mathrm{C} 10-\mathrm{H} 10 \cdots \mathrm{N} 1$ & 0.95 & 2.73 & $3.4009(19)$ & 128 \\
$\mathrm{C} 15-\mathrm{H} 15 A \cdots \mathrm{O} 3^{\mathrm{i}}$ & 0.99 & 2.57 & $3.2179(16)$ & 123 \\
$\mathrm{C} 11-\mathrm{H} 11 \cdots \mathrm{O}^{\mathrm{ii}}$ & 0.95 & 2.47 & $3.2446(17)$ & 138
\end{tabular}

Symmetry codes: (i) $-x+1,-y,-z+1$; (ii) $-x+2,-y+2,-z+1$.

2-Butoxy-1,4-dihydroxyanthraquinone (2)

Crystal data

$\mathrm{C}_{18} \mathrm{H}_{16} \mathrm{O}_{5}$

$M_{r}=312.31$

Monoclinic, $P 2_{1} / n$

$a=4.7730$ (9) $\AA$

$b=44.272(8) \AA$

$c=13.807(3) \AA$

$\beta=95.164(2)^{\circ}$

$V=2905.8(9) \AA^{3}$

$Z=8$
$F(000)=1312$

$D_{\mathrm{x}}=1.428 \mathrm{Mg} \mathrm{m}^{-3}$

Mo $K \alpha$ radiation, $\lambda=0.71073 \AA$

Cell parameters from 2921 reflections

$\theta=2.4-23.8^{\circ}$

$\mu=0.10 \mathrm{~mm}^{-1}$

$T=100 \mathrm{~K}$

Cut irregular block, orange-red

$0.48 \times 0.10 \times 0.03 \mathrm{~mm}$ 


\section{Data collection}

Bruker APEXII CCD

diffractometer

Radiation source: fine-focus sealed tube

Graphite monochromator

Detector resolution: 8.3660 pixels $\mathrm{mm}^{-1}$

phi and $\omega$ scans

Absorption correction: multi-scan

(SADABS; Bruker, 2014)

$T_{\min }=0.854, T_{\max }=1.000$

Refinement

Refinement on $F^{2}$

Least-squares matrix: full

$R\left[F^{2}>2 \sigma\left(F^{2}\right)\right]=0.063$

$w R\left(F^{2}\right)=0.166$

$S=1.04$

6456 reflections

429 parameters

4 restraints
36901 measured reflections

6456 independent reflections

3747 reflections with $I>2 \sigma(I)$

$R_{\text {int }}=0.104$

$\theta_{\max }=27.2^{\circ}, \theta_{\min }=1.6^{\circ}$

$h=-6 \rightarrow 6$

$k=-56 \rightarrow 56$

$l=-17 \rightarrow 17$

\section{Special details}

Geometry. All esds (except the esd in the dihedral angle between two 1.s. planes) are estimated using the full covariance matrix. The cell esds are taken into account individually in the estimation of esds in distances, angles and torsion angles; correlations between esds in cell parameters are only used when they are defined by crystal symmetry. An approximate (isotropic) treatment of cell esds is used for estimating esds involving l.s. planes.

Fractional atomic coordinates and isotropic or equivalent isotropic displacement parameters $\left(\hat{A}^{2}\right)$

\begin{tabular}{lllll}
\hline & $x$ & $y$ & $z$ & $U_{\text {iso }} / U_{\text {eq }}$ \\
\hline O1 & $-0.3186(4)$ & $0.27290(4)$ & $0.38526(14)$ & $0.0341(5)$ \\
$\mathrm{H} 1 \mathrm{O}$ & $-0.448(5)$ & $0.2616(6)$ & $0.359(2)$ & $0.041^{*}$ \\
$\mathrm{C} 1$ & $-0.3234(6)$ & $0.29688(6)$ & $0.32535(19)$ & $0.0272(6)$ \\
$\mathrm{O} 2$ & $0.0428(4)$ & $0.31529(4)$ & $0.43337(13)$ & $0.0312(5)$ \\
$\mathrm{C} 2$ & $-0.1245(6)$ & $0.32043(6)$ & $0.3504(2)$ & $0.0275(6)$ \\
$\mathrm{O} 3$ & $-0.2823(4)$ & $0.37359(4)$ & $0.15477(15)$ & $0.0350(5)$ \\
$\mathrm{H} 3 \mathrm{O}$ & $-0.399(6)$ & $0.3708(7)$ & $0.1034(17)$ & $0.042^{*}$ \\
$\mathrm{C} 3$ & $-0.1160(6)$ & $0.34561(6)$ & $0.2928(2)$ & $0.0287(6)$ \\
$\mathrm{H} 3$ & 0.017152 & 0.361103 & 0.309821 & $0.034 *$ \\
O4 & $-0.6827(4)$ & $0.35220(4)$ & $0.04208(14)$ & $0.0367(5)$ \\
$\mathrm{C} 4$ & $-0.3032(6)$ & $0.34858(6)$ & $0.2089(2)$ & $0.0286(6)$ \\
O5 & $-0.7188(4)$ & $0.25237(4)$ & $0.26716(15)$ & $0.0375(5)$ \\
$\mathrm{C} 5$ & $-0.5023(5)$ & $0.32611(6)$ & $0.18344(19)$ & $0.0254(6)$ \\
O6 & $0.7609(4)$ & $0.39771(4)$ & $0.48700(15)$ & $0.0355(5)$ \\
$\mathrm{H} 6 \mathrm{O}$ & $0.636(6)$ & $0.3937(7)$ & $0.4395(17)$ & $0.043 *$ \\
$\mathrm{C} 6$ & $-0.6920(6)$ & $0.32954(6)$ & $0.0958(2)$ & $0.0293(6)$ \\
O7 & $1.1866(4)$ & $0.42067(4)$ & $0.59154(14)$ & $0.0367(5)$ \\
C7 & $-0.9003(5)$ & $0.30521(6)$ & $0.07061(19)$ & $0.0266(6)$ \\
O8 & $1.2269(4)$ & $0.50371(4)$ & $0.37289(15)$ & $0.0341(5)$ \\
$\mathrm{H} 8 \mathrm{O}$ & $1.123(6)$ & $0.5094(7)$ & $0.3208(16)$ & $0.041^{*}$ \\
C8 & $-1.0870(6)$ & $0.30801(6)$ & $-0.0124(2)$ & $0.0328(7)$
\end{tabular}




\begin{tabular}{|c|c|c|c|c|}
\hline $\mathrm{H} 8$ & -1.081236 & 0.325412 & -0.052455 & $0.039^{*}$ \\
\hline O9 & $0.8632(4)$ & $0.50517(4)$ & $0.22553(14)$ & $0.0359(5)$ \\
\hline C9 & $-1.2818(6)$ & $0.28529(7)$ & $-0.0366(2)$ & $0.0349(7)$ \\
\hline H9 & -1.410599 & 0.287331 & -0.092840 & $0.042 *$ \\
\hline $\mathrm{O} 10$ & $0.4078(4)$ & $0.40025(4)$ & $0.33801(14)$ & $0.0346(5)$ \\
\hline $\mathrm{C} 10$ & $-1.2893(6)$ & $0.25958(6)$ & $0.0210(2)$ & $0.0340(7)$ \\
\hline $\mathrm{H} 10$ & -1.419875 & 0.243897 & 0.003368 & $0.041 *$ \\
\hline C11 & $-1.1065(6)$ & $0.25689(6)$ & $0.1040(2)$ & $0.0321(7)$ \\
\hline H11 & -1.114821 & 0.239564 & 0.144244 & $0.038^{*}$ \\
\hline $\mathrm{C} 12$ & $-0.9090(6)$ & $0.27958(6)$ & 0.12909 (19) & $0.0274(6)$ \\
\hline $\mathrm{C} 13$ & $-0.7114(6)$ & $0.27581(6)$ & $0.2171(2)$ & $0.0286(6)$ \\
\hline $\mathrm{C} 14$ & $-0.5100(5)$ & $0.29996(6)$ & $0.2427(2)$ & $0.0264(6)$ \\
\hline $\mathrm{C} 15$ & $0.2386(6)$ & $0.33909(6)$ & $0.4653(2)$ & $0.0313(6)$ \\
\hline H15B & 0.136313 & 0.358244 & 0.473426 & $0.038^{*}$ \\
\hline H15A & 0.375876 & 0.342303 & 0.416602 & $0.038 *$ \\
\hline $\mathrm{C} 16$ & $0.3885(6)$ & $0.32930(6)$ & $0.5611(2)$ & $0.0339(7)$ \\
\hline H16A & 0.538850 & 0.344065 & 0.580570 & $0.041 *$ \\
\hline H16B & 0.479215 & 0.309504 & 0.552161 & $0.041^{*}$ \\
\hline C17 & $0.1962(7)$ & $0.32659(7)$ & $0.6425(2)$ & $0.0402(7)$ \\
\hline H17A & 0.060155 & 0.310046 & 0.626768 & $0.048^{*}$ \\
\hline H17B & 0.088094 & 0.345572 & 0.646233 & $0.048^{*}$ \\
\hline $\mathrm{C} 18$ & $0.3532(8)$ & $0.32039(7)$ & $0.7413(2)$ & $0.0488(9)$ \\
\hline H18A & 0.475930 & 0.302797 & 0.736548 & $0.073^{*}$ \\
\hline H18B & 0.217716 & 0.316330 & 0.788912 & $0.073 *$ \\
\hline $\mathrm{H} 18 \mathrm{C}$ & 0.467288 & 0.338036 & 0.761934 & $0.073^{*}$ \\
\hline C19 & $0.8649(6)$ & $0.42371(6)$ & $0.4540(2)$ & $0.0296(6)$ \\
\hline $\mathrm{C} 20$ & $1.0975(6)$ & $0.43683(6)$ & $0.5119(2)$ & $0.0301(6)$ \\
\hline $\mathrm{C} 21$ & $1.2100(6)$ & $0.46359(6)$ & $0.4840(2)$ & $0.0302(6)$ \\
\hline $\mathrm{H} 21$ & 1.362242 & 0.472501 & 0.522992 & $0.036^{*}$ \\
\hline $\mathrm{C} 22$ & $1.1009(6)$ & $0.47784(6)$ & $0.3981(2)$ & $0.0300(6)$ \\
\hline $\mathrm{C} 23$ & $0.8739(6)$ & $0.46552(6)$ & $0.3399(2)$ & $0.0278(6)$ \\
\hline $\mathrm{C} 24$ & $0.7634(6)$ & $0.48070(6)$ & $0.2524(2)$ & $0.0293(6)$ \\
\hline $\mathrm{C} 25$ & $0.5272(6)$ & $0.46627(6)$ & $0.1918(2)$ & $0.0300(6)$ \\
\hline $\mathrm{C} 26$ & $0.4175(6)$ & $0.48005(7)$ & $0.1061(2)$ & $0.0365(7)$ \\
\hline $\mathrm{H} 26$ & 0.489850 & 0.498971 & 0.087551 & $0.044 *$ \\
\hline $\mathrm{C} 27$ & $0.2030(7)$ & $0.46627(7)$ & $0.0475(2)$ & $0.0413(8)$ \\
\hline $\mathrm{H} 27$ & 0.132225 & 0.475545 & -0.011761 & $0.050 *$ \\
\hline $\mathrm{C} 28$ & 0.0917 (6) & $0.43906(7)$ & $0.0752(2)$ & $0.0394(7)$ \\
\hline $\mathrm{H} 28$ & -0.057104 & 0.429841 & 0.035422 & $0.047^{*}$ \\
\hline C29 & $0.1970(6)$ & $0.42527(6)$ & $0.1608(2)$ & $0.0354(7)$ \\
\hline $\mathrm{H} 29$ & 0.120415 & 0.406580 & 0.179632 & $0.042 *$ \\
\hline C30 & $0.4146(6)$ & $0.43869(6)$ & $0.2195(2)$ & $0.0305(6)$ \\
\hline $\mathrm{C} 31$ & $0.5203(6)$ & $0.42406(6)$ & $0.3111(2)$ & $0.0296(6)$ \\
\hline $\mathrm{C} 32$ & $0.7571(6)$ & $0.43774(6)$ & $0.3698(2)$ & $0.0290(6)$ \\
\hline $\mathrm{C} 33$ & $1.4201(6)$ & $0.43232(6)$ & $0.6555(2)$ & $0.0354(7)$ \\
\hline H33A & 1.359002 & 0.449853 & 0.692861 & $0.042 *$ \\
\hline H33B & 1.575349 & 0.438816 & 0.617335 & $0.042 *$ \\
\hline C34 & $1.5157(7)$ & $0.40682(7)$ & $0.7235(2)$ & $0.0392(7)$ \\
\hline
\end{tabular}




\begin{tabular}{lllll} 
H34A & 1.589349 & 0.390176 & 0.685154 & $0.047^{*}$ \\
H34B & 1.351732 & 0.398995 & 0.754780 & $0.047^{*}$ \\
C35 & $1.7415(7)$ & $0.41641(7)$ & $0.8018(2)$ & $0.0449(8)$ \\
H35A & 1.663659 & 0.431719 & 0.844235 & $0.054^{*}$ \\
H35B & 1.900044 & 0.425732 & 0.771114 & $0.054^{*}$ \\
C36 & $1.8488(8)$ & $0.38931(8)$ & $0.8632(2)$ & $0.0525(9)$ \\
H36A & 1.695632 & 0.381183 & 0.898166 & $0.079^{*}$ \\
H36B & 2.003713 & 0.395739 & 0.910094 & $0.079^{*}$ \\
H36C & 1.915812 & 0.373660 & 0.820721 & $0.079^{*}$ \\
\hline
\end{tabular}

Atomic displacement parameters $\left(\AA^{2}\right)$

\begin{tabular}{|c|c|c|c|c|c|c|}
\hline & $U^{11}$ & $U^{22}$ & $U^{33}$ & $U^{12}$ & $U^{13}$ & $U^{23}$ \\
\hline $\mathrm{O} 1$ & $0.0317(11)$ & $0.0275(11)$ & $0.0412(12)$ & $-0.0051(9)$ & $-0.0072(9)$ & 0.0049 (9) \\
\hline $\mathrm{C} 1$ & $0.0237(14)$ & $0.0243(14)$ & $0.0334(15)$ & $0.0016(11)$ & $0.0025(12)$ & $0.0025(12)$ \\
\hline $\mathrm{O} 2$ & $0.0262(10)$ & $0.0271(10)$ & $0.0389(11)$ & $-0.0023(8)$ & $-0.0038(9)$ & $-0.0001(8)$ \\
\hline $\mathrm{C} 2$ & $0.0236(14)$ & $0.0239(14)$ & $0.0350(15)$ & $0.0027(11)$ & $0.0020(12)$ & $-0.0028(11)$ \\
\hline $\mathrm{O} 3$ & $0.0343(12)$ & $0.0276(11)$ & $0.0423(12)$ & $-0.0038(9)$ & $-0.0007(9)$ & $0.0058(9)$ \\
\hline $\mathrm{C} 3$ & $0.0229(14)$ & $0.0232(14)$ & $0.0400(16)$ & $-0.0018(11)$ & $0.0033(12)$ & $-0.0031(12)$ \\
\hline $\mathrm{O} 4$ & $0.0353(12)$ & $0.0298(11)$ & $0.0446(12)$ & $0.0010(9)$ & $0.0009(9)$ & $0.0078(9)$ \\
\hline $\mathrm{C} 4$ & $0.0251(15)$ & $0.0226(14)$ & $0.0387(16)$ & $0.0024(11)$ & $0.0057(12)$ & $0.0020(12)$ \\
\hline $\mathrm{O} 5$ & $0.0347(12)$ & $0.0297(11)$ & $0.0464(12)$ & $-0.0070(9)$ & $-0.0060(9)$ & $0.0081(9)$ \\
\hline $\mathrm{C} 5$ & $0.0211(14)$ & $0.0233(13)$ & $0.0325(15)$ & $0.0019(11)$ & $0.0059(11)$ & $-0.0008(11)$ \\
\hline O6 & $0.0331(12)$ & $0.0261(10)$ & $0.0468(13)$ & $-0.0057(9)$ & $0.0015(9)$ & $0.0027(9)$ \\
\hline C6 & $0.0236(14)$ & $0.0279(15)$ & $0.0364(16)$ & $0.0066(11)$ & $0.0024(12)$ & $0.0021(12)$ \\
\hline $\mathrm{O} 7$ & $0.0321(11)$ & $0.0302(11)$ & $0.0467(12)$ & $-0.0039(9)$ & $-0.0022(9)$ & $0.0032(9)$ \\
\hline $\mathrm{C} 7$ & $0.0224(14)$ & $0.0272(14)$ & $0.0306(15)$ & $0.0048(11)$ & $0.0048(12)$ & $-0.0013(11)$ \\
\hline $\mathrm{O} 8$ & $0.0304(11)$ & $0.0261(10)$ & $0.0456(12)$ & $-0.0053(9)$ & $0.0028(9)$ & $0.0013(9)$ \\
\hline $\mathrm{C} 8$ & $0.0322(16)$ & $0.0337(16)$ & $0.0324(16)$ & $0.0068(13)$ & $0.0024(13)$ & $0.0022(12)$ \\
\hline O9 & $0.0342(12)$ & $0.0263(11)$ & $0.0474(12)$ & $-0.0037(9)$ & 0.0047 (9) & $0.0027(9)$ \\
\hline $\mathrm{C} 9$ & $0.0273(16)$ & $0.0401(17)$ & $0.0360(17)$ & $0.0071(13)$ & $-0.0035(13)$ & $-0.0072(13)$ \\
\hline $\mathrm{O} 10$ & $0.0291(11)$ & $0.0263(11)$ & $0.0487(12)$ & $-0.0046(8)$ & 0.0049 (9) & $-0.0003(9)$ \\
\hline $\mathrm{C} 10$ & $0.0279(16)$ & $0.0323(16)$ & $0.0411(17)$ & $-0.0001(12)$ & $-0.0006(13)$ & $-0.0099(13)$ \\
\hline C11 & $0.0292(16)$ & $0.0276(15)$ & $0.0394(16)$ & $-0.0003(12)$ & $0.0030(13)$ & $-0.0039(12)$ \\
\hline $\mathrm{C} 12$ & $0.0235(14)$ & $0.0266(14)$ & $0.0323(15)$ & $0.0036(11)$ & $0.0029(12)$ & $-0.0039(12)$ \\
\hline $\mathrm{C} 13$ & $0.0239(14)$ & $0.0282(15)$ & $0.0335(15)$ & $0.0013(12)$ & $0.0008(12)$ & $-0.0015(12)$ \\
\hline C14 & $0.0207(13)$ & $0.0232(14)$ & $0.0355(15)$ & $0.0006(11)$ & $0.0033(11)$ & $-0.0004(11)$ \\
\hline $\mathrm{C} 15$ & $0.0266(15)$ & $0.0225(14)$ & $0.0443(17)$ & $-0.0034(11)$ & 0.0009 (13) & $-0.0019(12)$ \\
\hline $\mathrm{C} 16$ & $0.0295(16)$ & $0.0277(15)$ & $0.0427(17)$ & $-0.0001(12)$ & $-0.0065(13)$ & $-0.0047(13)$ \\
\hline $\mathrm{C} 17$ & $0.0374(18)$ & $0.0405(18)$ & $0.0415(18)$ & $0.0057(14)$ & $-0.0024(14)$ & $-0.0005(14)$ \\
\hline $\mathrm{C} 18$ & $0.061(2)$ & $0.0373(19)$ & $0.0463(19)$ & $0.0090(16)$ & $-0.0058(17)$ & $-0.0034(15)$ \\
\hline C19 & $0.0277(15)$ & $0.0196(13)$ & $0.0425(17)$ & $-0.0021(11)$ & $0.0083(13)$ & $-0.0014(12)$ \\
\hline $\mathrm{C} 20$ & $0.0263(15)$ & $0.0263(14)$ & $0.0382(16)$ & $0.0031(12)$ & $0.0058(13)$ & $-0.0011(12)$ \\
\hline $\mathrm{C} 21$ & $0.0243(15)$ & 0.0255 (14) & $0.0412(17)$ & -0.0009 (11) & $0.0058(13)$ & $-0.0041(12)$ \\
\hline $\mathrm{C} 22$ & $0.0265(15)$ & $0.0216(14)$ & $0.0432(17)$ & $-0.0018(11)$ & $0.0107(13)$ & $-0.0056(12)$ \\
\hline $\mathrm{C} 23$ & $0.0235(14)$ & $0.0217(14)$ & $0.0386(16)$ & $0.0013(11)$ & $0.0058(12)$ & $-0.0025(12)$ \\
\hline C24 & $0.0236(14)$ & $0.0238(14)$ & $0.0413(16)$ & $0.0001(11)$ & $0.0069(12)$ & $-0.0037(12)$ \\
\hline $\mathrm{C} 25$ & $0.0247(15)$ & $0.0257(14)$ & $0.0404(16)$ & $0.0012(11)$ & $0.0064(12)$ & $-0.0027(12)$ \\
\hline
\end{tabular}


supporting information

\begin{tabular}{lllllll}
\hline & & & & & \\
C26 & $0.0330(17)$ & $0.0319(16)$ & $0.0445(18)$ & $-0.0002(13)$ & $0.0029(14)$ & $0.0034(13)$ \\
C27 & $0.0371(18)$ & $0.0395(18)$ & $0.0462(19)$ & $0.0042(14)$ & $-0.0024(15)$ & $0.0013(14)$ \\
C28 & $0.0311(17)$ & $0.0384(17)$ & $0.0475(19)$ & $0.0008(13)$ & $-0.0022(14)$ & $-0.0066(14)$ \\
C29 & $0.0277(16)$ & $0.0303(16)$ & $0.0483(18)$ & $-0.0006(12)$ & $0.0049(14)$ & $-0.0055(13)$ \\
C30 & $0.0240(15)$ & $0.0278(15)$ & $0.0401(17)$ & $0.0020(11)$ & $0.0055(13)$ & $-0.0051(12)$ \\
C31 & $0.0250(15)$ & $0.0234(14)$ & $0.0416(17)$ & $0.0023(11)$ & $0.0095(13)$ & $-0.0047(12)$ \\
C32 & $0.0227(14)$ & $0.0242(14)$ & $0.0405(16)$ & $0.0004(11)$ & $0.0059(12)$ & $-0.0039(12)$ \\
C33 & $0.0313(16)$ & $0.0294(15)$ & $0.0446(18)$ & $-0.0043(13)$ & $-0.0009(13)$ & $-0.0040(13)$ \\
C34 & $0.0383(18)$ & $0.0347(17)$ & $0.0440(18)$ & $0.0003(14)$ & $-0.0001(14)$ & $0.0010(14)$ \\
C35 & $0.046(2)$ & $0.0380(18)$ & $0.0495(19)$ & $0.0035(15)$ & $-0.0053(16)$ & $-0.0038(15)$ \\
C36 & $0.056(2)$ & $0.047(2)$ & $0.052(2)$ & $0.0065(17)$ & $-0.0088(17)$ & $-0.0009(16)$ \\
& & & & & & \\
\hline
\end{tabular}

Geometric parameters $\left(\AA,{ }^{o}\right)$

\begin{tabular}{|c|c|c|c|}
\hline $\mathrm{O} 1-\mathrm{C} 1$ & $1.345(3)$ & $\mathrm{C} 16-\mathrm{H} 16 \mathrm{~A}$ & 0.9900 \\
\hline $\mathrm{O} 1-\mathrm{H} 1 \mathrm{O}$ & $0.848(18)$ & C16-H16B & 0.9900 \\
\hline $\mathrm{C} 1-\mathrm{C} 14$ & $1.390(4)$ & $\mathrm{C} 17-\mathrm{C} 18$ & $1.520(4)$ \\
\hline $\mathrm{C} 1-\mathrm{C} 2$ & $1.431(4)$ & $\mathrm{C} 17-\mathrm{H} 17 \mathrm{~A}$ & 0.9900 \\
\hline $\mathrm{O} 2-\mathrm{C} 2$ & $1.356(3)$ & C17-H17B & 0.9900 \\
\hline $\mathrm{O} 2-\mathrm{C} 15$ & $1.450(3)$ & C18-H18A & 0.9800 \\
\hline $\mathrm{C} 2-\mathrm{C} 3$ & $1.372(4)$ & $\mathrm{C} 18-\mathrm{H} 18 \mathrm{~B}$ & 0.9800 \\
\hline $\mathrm{O} 3-\mathrm{C} 4$ & $1.344(3)$ & $\mathrm{C} 18-\mathrm{H} 18 \mathrm{C}$ & 0.9800 \\
\hline $\mathrm{O} 3-\mathrm{H} 3 \mathrm{O}$ & $0.870(17)$ & $\mathrm{C} 19-\mathrm{C} 32$ & $1.377(4)$ \\
\hline $\mathrm{C} 3-\mathrm{C} 4$ & $1.404(4)$ & $\mathrm{C} 19-\mathrm{C} 20$ & $1.432(4)$ \\
\hline $\mathrm{C} 3-\mathrm{H} 3$ & 0.9500 & $\mathrm{C} 20-\mathrm{C} 21$ & $1.370(4)$ \\
\hline $\mathrm{O} 4-\mathrm{C} 6$ & $1.251(3)$ & $\mathrm{C} 21-\mathrm{C} 22$ & $1.402(4)$ \\
\hline $\mathrm{C} 4-\mathrm{C} 5$ & $1.399(4)$ & $\mathrm{C} 21-\mathrm{H} 21$ & 0.9500 \\
\hline $\mathrm{O} 5-\mathrm{C} 13$ & $1.249(3)$ & $\mathrm{C} 22-\mathrm{C} 23$ & $1.400(4)$ \\
\hline $\mathrm{C} 5-\mathrm{C} 14$ & $1.420(4)$ & $\mathrm{C} 23-\mathrm{C} 32$ & $1.426(4)$ \\
\hline $\mathrm{C} 5-\mathrm{C} 6$ & $1.453(4)$ & $\mathrm{C} 23-\mathrm{C} 24$ & $1.441(4)$ \\
\hline O6- & $1.349(3)$ & $\mathrm{C} 24-\mathrm{C} 25$ & $1.486(4)$ \\
\hline $\mathrm{O} 6-\mathrm{H} 6 \mathrm{O}$ & $0.863(18)$ & $\mathrm{C} 25-\mathrm{C} 26$ & $1.392(4)$ \\
\hline $\mathrm{C} 6-\mathrm{C} 7$ & $1.485(4)$ & $\mathrm{C} 25-\mathrm{C} 30$ & $1.401(4)$ \\
\hline $\mathrm{O} 7-\mathrm{C} 20$ & $1.348(3)$ & $\mathrm{C} 26-\mathrm{C} 27$ & $1.388(4)$ \\
\hline $\mathrm{O} 7-\mathrm{C} 33$ & $1.453(3)$ & $\mathrm{C} 26-\mathrm{H} 26$ & 0.9500 \\
\hline $\mathrm{C} 7-\mathrm{C} 8$ & $1.393(4)$ & $\mathrm{C} 27-\mathrm{C} 28$ & $1.384(4)$ \\
\hline $\mathrm{C} 7-\mathrm{C} 12$ & $1.396(4)$ & $\mathrm{C} 27-\mathrm{H} 27$ & 0.9500 \\
\hline $\mathrm{O} 8-\mathrm{C} 22$ & $1.354(3)$ & $\mathrm{C} 28-\mathrm{C} 29$ & $1.384(4)$ \\
\hline $\mathrm{O} 8-\mathrm{H} 8 \mathrm{O}$ & $0.874(17)$ & $\mathrm{C} 28-\mathrm{H} 28$ & 0.9500 \\
\hline $\mathrm{C} 8-\mathrm{C} 9$ & $1.390(4)$ & $\mathrm{C} 29-\mathrm{C} 30$ & $1.392(4)$ \\
\hline $\mathrm{C} 8-\mathrm{H} 8$ & 0.9500 & $\mathrm{C} 29-\mathrm{H} 29$ & 0.9500 \\
\hline $\mathrm{O} 9-\mathrm{C} 24$ & $1.253(3)$ & $\mathrm{C} 30-\mathrm{C} 31$ & 1.469 (4) \\
\hline $\mathrm{C} 9-\mathrm{C} 10$ & $1.391(4)$ & $\mathrm{C} 31-\mathrm{C} 32$ & $1.462(4)$ \\
\hline C9- $\mathrm{H} 9$ & 0.9500 & $\mathrm{C} 33-\mathrm{C} 34$ & $1.512(4)$ \\
\hline $\mathrm{O} 10-\mathrm{C} 31$ & $1.254(3)$ & $\mathrm{C} 33-\mathrm{H} 33 \mathrm{~A}$ & 0.9900 \\
\hline $\mathrm{C} 10-\mathrm{C} 11$ & $1.381(4)$ & $\mathrm{C} 33-\mathrm{H} 33 \mathrm{~B}$ & 0.9900 \\
\hline $\mathrm{C} 10-\mathrm{H} 10$ & 0.9500 & C34-C35 & $1.517(4)$ \\
\hline $\mathrm{C} 11-\mathrm{C} 12$ & $1.399(4)$ & $\mathrm{C} 34-\mathrm{H} 34 \mathrm{~A}$ & 0.9900 \\
\hline
\end{tabular}




\begin{tabular}{|c|c|c|c|}
\hline $\mathrm{C} 11-\mathrm{H} 11$ & 0.9500 & $\mathrm{C} 34-\mathrm{H} 34 \mathrm{~B}$ & 0.9900 \\
\hline $\mathrm{C} 12-\mathrm{C} 13$ & $1.479(4)$ & $\mathrm{C} 35-\mathrm{C} 36$ & $1.531(4)$ \\
\hline $\mathrm{C} 13-\mathrm{C} 14$ & $1.460(4)$ & $\mathrm{C} 35-\mathrm{H} 35 \mathrm{~A}$ & 0.9900 \\
\hline $\mathrm{C} 15-\mathrm{C} 16$ & $1.510(4)$ & C $35-\mathrm{H} 35 \mathrm{~B}$ & 0.9900 \\
\hline C15-H15B & 0.9900 & $\mathrm{C} 36-\mathrm{H} 36 \mathrm{~A}$ & 0.9800 \\
\hline C15-H15A & 0.9900 & $\mathrm{C} 36-\mathrm{H} 36 \mathrm{~B}$ & 0.9800 \\
\hline $\mathrm{C} 16-\mathrm{C} 17$ & $1.518(4)$ & $\mathrm{C} 36-\mathrm{H} 36 \mathrm{C}$ & 0.9800 \\
\hline $\mathrm{C} 1-\mathrm{O} 1-\mathrm{H} 1 \mathrm{O}$ & $103(2)$ & $\mathrm{C} 17-\mathrm{C} 18-\mathrm{H} 18 \mathrm{C}$ & 109.5 \\
\hline $\mathrm{O} 1-\mathrm{C} 1-\mathrm{C} 14$ & $123.8(2)$ & $\mathrm{H} 18 \mathrm{~A}-\mathrm{C} 18-\mathrm{H} 18 \mathrm{C}$ & 109.5 \\
\hline $\mathrm{O} 1-\mathrm{C} 1-\mathrm{C} 2$ & $116.9(2)$ & $\mathrm{H} 18 \mathrm{~B}-\mathrm{C} 18-\mathrm{H} 18 \mathrm{C}$ & 109.5 \\
\hline $\mathrm{C} 14-\mathrm{C} 1-\mathrm{C} 2$ & $119.3(2)$ & $\mathrm{O} 6-\mathrm{C} 19-\mathrm{C} 32$ & $123.3(2)$ \\
\hline $\mathrm{C} 2-\mathrm{O} 2-\mathrm{C} 15$ & $116.7(2)$ & $\mathrm{O} 6-\mathrm{C} 19-\mathrm{C} 20$ & $116.6(2)$ \\
\hline $\mathrm{O} 2-\mathrm{C} 2-\mathrm{C} 3$ & $125.4(2)$ & $\mathrm{C} 32-\mathrm{C} 19-\mathrm{C} 20$ & $120.1(2)$ \\
\hline $\mathrm{O} 2-\mathrm{C} 2-\mathrm{C} 1$ & $114.2(2)$ & $\mathrm{O} 7-\mathrm{C} 20-\mathrm{C} 21$ & $125.8(3)$ \\
\hline $\mathrm{C} 3-\mathrm{C} 2-\mathrm{C} 1$ & $120.4(2)$ & $\mathrm{O} 7-\mathrm{C} 20-\mathrm{C} 19$ & $114.4(2)$ \\
\hline $\mathrm{C} 4-\mathrm{O} 3-\mathrm{H} 3 \mathrm{O}$ & $105(2)$ & $\mathrm{C} 21-\mathrm{C} 20-\mathrm{C} 19$ & $119.9(3)$ \\
\hline $\mathrm{C} 2-\mathrm{C} 3-\mathrm{C} 4$ & $120.3(2)$ & $\mathrm{C} 20-\mathrm{C} 21-\mathrm{C} 22$ & $120.1(3)$ \\
\hline $\mathrm{C} 2-\mathrm{C} 3-\mathrm{H} 3$ & 119.9 & $\mathrm{C} 20-\mathrm{C} 21-\mathrm{H} 21$ & 120.0 \\
\hline $\mathrm{C} 4-\mathrm{C} 3-\mathrm{H} 3$ & 119.9 & $\mathrm{C} 22-\mathrm{C} 21-\mathrm{H} 21$ & 120.0 \\
\hline $\mathrm{O} 3-\mathrm{C} 4-\mathrm{C} 5$ & $121.9(2)$ & $\mathrm{O} 8-\mathrm{C} 22-\mathrm{C} 23$ & $121.4(3)$ \\
\hline $\mathrm{O} 3-\mathrm{C} 4-\mathrm{C} 3$ & $117.5(2)$ & $\mathrm{O} 8-\mathrm{C} 22-\mathrm{C} 21$ & $117.3(2)$ \\
\hline $\mathrm{C} 5-\mathrm{C} 4-\mathrm{C} 3$ & $120.5(2)$ & $\mathrm{C} 23-\mathrm{C} 22-\mathrm{C} 21$ & $121.3(2)$ \\
\hline $\mathrm{C} 4-\mathrm{C} 5-\mathrm{C} 14$ & $119.2(2)$ & $\mathrm{C} 22-\mathrm{C} 23-\mathrm{C} 32$ & $118.2(2)$ \\
\hline $\mathrm{C} 4-\mathrm{C} 5-\mathrm{C} 6$ & $119.6(2)$ & $\mathrm{C} 22-\mathrm{C} 23-\mathrm{C} 24$ & $120.4(2)$ \\
\hline $\mathrm{C} 14-\mathrm{C} 5-\mathrm{C} 6$ & $121.2(2)$ & $\mathrm{C} 32-\mathrm{C} 23-\mathrm{C} 24$ & $121.4(2)$ \\
\hline $\mathrm{C} 19-\mathrm{O} 6-\mathrm{H} 6 \mathrm{O}$ & $100(2)$ & $\mathrm{O} 9-\mathrm{C} 24-\mathrm{C} 23$ & $122.1(3)$ \\
\hline $\mathrm{O} 4-\mathrm{C} 6-\mathrm{C} 5$ & $121.7(2)$ & $\mathrm{O} 9-\mathrm{C} 24-\mathrm{C} 25$ & $119.7(3)$ \\
\hline $\mathrm{O} 4-\mathrm{C} 6-\mathrm{C} 7$ & $120.1(2)$ & $\mathrm{C} 23-\mathrm{C} 24-\mathrm{C} 25$ & $118.2(2)$ \\
\hline $\mathrm{C} 5-\mathrm{C} 6-\mathrm{C} 7$ & $118.2(2)$ & $\mathrm{C} 26-\mathrm{C} 25-\mathrm{C} 30$ & $119.3(3)$ \\
\hline $\mathrm{C} 20-\mathrm{O} 7-\mathrm{C} 33$ & $118.4(2)$ & $\mathrm{C} 26-\mathrm{C} 25-\mathrm{C} 24$ & $119.9(3)$ \\
\hline $\mathrm{C} 8-\mathrm{C} 7-\mathrm{C} 12$ & $119.9(3)$ & $\mathrm{C} 30-\mathrm{C} 25-\mathrm{C} 24$ & $120.8(3)$ \\
\hline $\mathrm{C} 8-\mathrm{C} 7-\mathrm{C} 6$ & $119.6(2)$ & $\mathrm{C} 27-\mathrm{C} 26-\mathrm{C} 25$ & $120.3(3)$ \\
\hline $\mathrm{C} 12-\mathrm{C} 7-\mathrm{C} 6$ & $120.6(2)$ & $\mathrm{C} 27-\mathrm{C} 26-\mathrm{H} 26$ & 119.8 \\
\hline $\mathrm{C} 22-\mathrm{O} 8-\mathrm{H} 8 \mathrm{O}$ & $103(2)$ & $\mathrm{C} 25-\mathrm{C} 26-\mathrm{H} 26$ & 119.8 \\
\hline $\mathrm{C} 9-\mathrm{C} 8-\mathrm{C} 7$ & $119.9(3)$ & $\mathrm{C} 28-\mathrm{C} 27-\mathrm{C} 26$ & $120.2(3)$ \\
\hline $\mathrm{C} 9-\mathrm{C} 8-\mathrm{H} 8$ & 120.1 & $\mathrm{C} 28-\mathrm{C} 27-\mathrm{H} 27$ & 119.9 \\
\hline $\mathrm{C} 7-\mathrm{C} 8-\mathrm{H} 8$ & 120.1 & $\mathrm{C} 26-\mathrm{C} 27-\mathrm{H} 27$ & 119.9 \\
\hline $\mathrm{C} 8-\mathrm{C} 9-\mathrm{C} 10$ & $120.4(3)$ & $\mathrm{C} 27-\mathrm{C} 28-\mathrm{C} 29$ & $120.1(3)$ \\
\hline $\mathrm{C} 8-\mathrm{C} 9-\mathrm{H} 9$ & 119.8 & $\mathrm{C} 27-\mathrm{C} 28-\mathrm{H} 28$ & 120.0 \\
\hline $\mathrm{C} 10-\mathrm{C} 9-\mathrm{H} 9$ & 119.8 & $\mathrm{C} 29-\mathrm{C} 28-\mathrm{H} 28$ & 120.0 \\
\hline $\mathrm{C} 11-\mathrm{C} 10-\mathrm{C} 9$ & $119.9(3)$ & $\mathrm{C} 28-\mathrm{C} 29-\mathrm{C} 30$ & $120.2(3)$ \\
\hline $\mathrm{C} 11-\mathrm{C} 10-\mathrm{H} 10$ & 120.1 & $\mathrm{C} 28-\mathrm{C} 29-\mathrm{H} 29$ & 119.9 \\
\hline C9-C10-H10 & 120.1 & $\mathrm{C} 30-\mathrm{C} 29-\mathrm{H} 29$ & 119.9 \\
\hline $\mathrm{C} 10-\mathrm{C} 11-\mathrm{C} 12$ & $120.3(3)$ & $\mathrm{C} 29-\mathrm{C} 30-\mathrm{C} 25$ & $119.9(3)$ \\
\hline $\mathrm{C} 10-\mathrm{C} 11-\mathrm{H} 11$ & 119.9 & $\mathrm{C} 29-\mathrm{C} 30-\mathrm{C} 31$ & $119.5(3)$ \\
\hline $\mathrm{C} 12-\mathrm{C} 11-\mathrm{H} 11$ & 119.9 & $\mathrm{C} 25-\mathrm{C} 30-\mathrm{C} 31$ & $120.6(3)$ \\
\hline $\mathrm{C} 7-\mathrm{C} 12-\mathrm{C} 11$ & $119.7(2)$ & $\mathrm{O} 10-\mathrm{C} 31-\mathrm{C} 32$ & $121.0(3)$ \\
\hline
\end{tabular}




\begin{tabular}{|c|c|c|c|}
\hline $\mathrm{C} 7-\mathrm{C} 12-\mathrm{C} 13$ & $121.1(2)$ & $\mathrm{O} 10-\mathrm{C} 31-\mathrm{C} 30$ & $120.2(2)$ \\
\hline $\mathrm{C} 11-\mathrm{C} 12-\mathrm{C} 13$ & $119.2(2)$ & $\mathrm{C} 32-\mathrm{C} 31-\mathrm{C} 30$ & $118.9(2)$ \\
\hline $\mathrm{O} 5-\mathrm{C} 13-\mathrm{C} 14$ & $121.7(2)$ & $\mathrm{C} 19-\mathrm{C} 32-\mathrm{C} 23$ & $120.5(2)$ \\
\hline $\mathrm{O} 5-\mathrm{C} 13-\mathrm{C} 12$ & $120.0(2)$ & $\mathrm{C} 19-\mathrm{C} 32-\mathrm{C} 31$ & $119.5(2)$ \\
\hline $\mathrm{C} 14-\mathrm{C} 13-\mathrm{C} 12$ & $118.2(2)$ & $\mathrm{C} 23-\mathrm{C} 32-\mathrm{C} 31$ & $120.0(2)$ \\
\hline $\mathrm{C} 1-\mathrm{C} 14-\mathrm{C} 5$ & $120.3(2)$ & $\mathrm{O} 7-\mathrm{C} 33-\mathrm{C} 34$ & $106.5(2)$ \\
\hline $\mathrm{C} 1-\mathrm{C} 14-\mathrm{C} 13$ & $119.1(2)$ & $\mathrm{O} 7-\mathrm{C} 33-\mathrm{H} 33 \mathrm{~A}$ & 110.4 \\
\hline $\mathrm{C} 5-\mathrm{C} 14-\mathrm{C} 13$ & $120.6(2)$ & $\mathrm{C} 34-\mathrm{C} 33-\mathrm{H} 33 \mathrm{~A}$ & 110.4 \\
\hline $\mathrm{O} 2-\mathrm{C} 15-\mathrm{C} 16$ & $107.4(2)$ & O7-C33-H33B & 110.4 \\
\hline $\mathrm{O} 2-\mathrm{C} 15-\mathrm{H} 15 \mathrm{~B}$ & 110.2 & $\mathrm{C} 34-\mathrm{C} 33-\mathrm{H} 33 \mathrm{~B}$ & 110.4 \\
\hline $\mathrm{C} 16-\mathrm{C} 15-\mathrm{H} 15 \mathrm{~B}$ & 110.2 & $\mathrm{H} 33 \mathrm{~A}-\mathrm{C} 33-\mathrm{H} 33 \mathrm{~B}$ & 108.6 \\
\hline $\mathrm{O} 2-\mathrm{C} 15-\mathrm{H} 15 \mathrm{~A}$ & 110.2 & $\mathrm{C} 33-\mathrm{C} 34-\mathrm{C} 35$ & $112.9(2)$ \\
\hline $\mathrm{C} 16-\mathrm{C} 15-\mathrm{H} 15 \mathrm{~A}$ & 110.2 & $\mathrm{C} 33-\mathrm{C} 34-\mathrm{H} 34 \mathrm{~A}$ & 109.0 \\
\hline $\mathrm{H} 15 \mathrm{~B}-\mathrm{C} 15-\mathrm{H} 15 \mathrm{~A}$ & 108.5 & $\mathrm{C} 35-\mathrm{C} 34-\mathrm{H} 34 \mathrm{~A}$ & 109.0 \\
\hline $\mathrm{C} 15-\mathrm{C} 16-\mathrm{C} 17$ & $113.7(2)$ & $\mathrm{C} 33-\mathrm{C} 34-\mathrm{H} 34 \mathrm{~B}$ & 109.0 \\
\hline $\mathrm{C} 15-\mathrm{C} 16-\mathrm{H} 16 \mathrm{~A}$ & 108.8 & С $35-\mathrm{C} 34-\mathrm{H} 34 \mathrm{~B}$ & 109.0 \\
\hline $\mathrm{C} 17-\mathrm{C} 16-\mathrm{H} 16 \mathrm{~A}$ & 108.8 & $\mathrm{H} 34 \mathrm{~A}-\mathrm{C} 34-\mathrm{H} 34 \mathrm{~B}$ & 107.8 \\
\hline $\mathrm{C} 15-\mathrm{C} 16-\mathrm{H} 16 \mathrm{~B}$ & 108.8 & $\mathrm{C} 34-\mathrm{C} 35-\mathrm{C} 36$ & $110.9(3)$ \\
\hline $\mathrm{C} 17-\mathrm{C} 16-\mathrm{H} 16 \mathrm{~B}$ & 108.8 & $\mathrm{C} 34-\mathrm{C} 35-\mathrm{H} 35 \mathrm{~A}$ & 109.5 \\
\hline $\mathrm{H} 16 \mathrm{~A}-\mathrm{C} 16-\mathrm{H} 16 \mathrm{~B}$ & 107.7 & $\mathrm{C} 36-\mathrm{C} 35-\mathrm{H} 35 \mathrm{~A}$ & 109.5 \\
\hline $\mathrm{C} 16-\mathrm{C} 17-\mathrm{C} 18$ & $113.3(3)$ & $\mathrm{C} 34-\mathrm{C} 35-\mathrm{H} 35 \mathrm{~B}$ & 109.5 \\
\hline $\mathrm{C} 16-\mathrm{C} 17-\mathrm{H} 17 \mathrm{~A}$ & 108.9 & $\mathrm{C} 36-\mathrm{C} 35-\mathrm{H} 35 \mathrm{~B}$ & 109.5 \\
\hline $\mathrm{C} 18-\mathrm{C} 17-\mathrm{H} 17 \mathrm{~A}$ & 108.9 & $\mathrm{H} 35 \mathrm{~A}-\mathrm{C} 35-\mathrm{H} 35 \mathrm{~B}$ & 108.1 \\
\hline $\mathrm{C} 16-\mathrm{C} 17-\mathrm{H} 17 \mathrm{~B}$ & 108.9 & $\mathrm{C} 35-\mathrm{C} 36-\mathrm{H} 36 \mathrm{~A}$ & 109.5 \\
\hline C18-C17-H17B & 108.9 & $\mathrm{C} 35-\mathrm{C} 36-\mathrm{H} 36 \mathrm{~B}$ & 109.5 \\
\hline $\mathrm{H} 17 \mathrm{~A}-\mathrm{C} 17-\mathrm{H} 17 \mathrm{~B}$ & 107.7 & $\mathrm{H} 36 \mathrm{~A}-\mathrm{C} 36-\mathrm{H} 36 \mathrm{~B}$ & 109.5 \\
\hline $\mathrm{C} 17-\mathrm{C} 18-\mathrm{H} 18 \mathrm{~A}$ & 109.5 & $\mathrm{C} 35-\mathrm{C} 36-\mathrm{H} 36 \mathrm{C}$ & 109.5 \\
\hline $\mathrm{C} 17-\mathrm{C} 18-\mathrm{H} 18 \mathrm{~B}$ & 109.5 & $\mathrm{H} 36 \mathrm{~A}-\mathrm{C} 36-\mathrm{H} 36 \mathrm{C}$ & 109.5 \\
\hline $\mathrm{H} 18 \mathrm{~A}-\mathrm{C} 18-\mathrm{H} 18 \mathrm{~B}$ & 109.5 & $\mathrm{H} 36 \mathrm{~B}-\mathrm{C} 36-\mathrm{H} 36 \mathrm{C}$ & 109.5 \\
\hline $\mathrm{C} 15-\mathrm{O} 2-\mathrm{C} 2-\mathrm{C} 3$ & $2.6(4)$ & $\mathrm{C} 33-\mathrm{O} 7-\mathrm{C} 20-\mathrm{C} 21$ & $0.9(4)$ \\
\hline $\mathrm{C} 15-\mathrm{O} 2-\mathrm{C} 2-\mathrm{C} 1$ & $-176.8(2)$ & $\mathrm{C} 33-\mathrm{O} 7-\mathrm{C} 20-\mathrm{C} 19$ & $-179.4(2)$ \\
\hline $\mathrm{O} 1-\mathrm{C} 1-\mathrm{C} 2-\mathrm{O} 2$ & $-0.6(3)$ & $\mathrm{O} 6-\mathrm{C} 19-\mathrm{C} 20-\mathrm{O} 7$ & $1.7(4)$ \\
\hline $\mathrm{C} 14-\mathrm{C} 1-\mathrm{C} 2-\mathrm{O} 2$ & $178.7(2)$ & $\mathrm{C} 32-\mathrm{C} 19-\mathrm{C} 20-\mathrm{O} 7$ & $-178.9(2)$ \\
\hline $\mathrm{O} 1-\mathrm{C} 1-\mathrm{C} 2-\mathrm{C} 3$ & $179.9(2)$ & $\mathrm{O} 6-\mathrm{C} 19-\mathrm{C} 20-\mathrm{C} 21$ & $-178.5(2)$ \\
\hline $\mathrm{C} 14-\mathrm{C} 1-\mathrm{C} 2-\mathrm{C} 3$ & $-0.7(4)$ & $\mathrm{C} 32-\mathrm{C} 19-\mathrm{C} 20-\mathrm{C} 21$ & $0.8(4)$ \\
\hline $\mathrm{O} 2-\mathrm{C} 2-\mathrm{C} 3-\mathrm{C} 4$ & $-179.2(2)$ & $\mathrm{O} 7-\mathrm{C} 20-\mathrm{C} 21-\mathrm{C} 22$ & $178.6(3)$ \\
\hline $\mathrm{C} 1-\mathrm{C} 2-\mathrm{C} 3-\mathrm{C} 4$ & $0.2(4)$ & $\mathrm{C} 19-\mathrm{C} 20-\mathrm{C} 21-\mathrm{C} 22$ & $-1.1(4)$ \\
\hline $\mathrm{C} 2-\mathrm{C} 3-\mathrm{C} 4-\mathrm{O} 3$ & $-179.2(2)$ & $\mathrm{C} 20-\mathrm{C} 21-\mathrm{C} 22-\mathrm{O} 8$ & $-177.9(2)$ \\
\hline $\mathrm{C} 2-\mathrm{C} 3-\mathrm{C} 4-\mathrm{C} 5$ & $0.6(4)$ & $\mathrm{C} 20-\mathrm{C} 21-\mathrm{C} 22-\mathrm{C} 23$ & $1.0(4)$ \\
\hline $\mathrm{O} 3-\mathrm{C} 4-\mathrm{C} 5-\mathrm{C} 14$ & $178.8(2)$ & $\mathrm{O} 8-\mathrm{C} 22-\mathrm{C} 23-\mathrm{C} 32$ & $178.2(2)$ \\
\hline $\mathrm{C} 3-\mathrm{C} 4-\mathrm{C} 5-\mathrm{C} 14$ & $-1.0(4)$ & $\mathrm{C} 21-\mathrm{C} 22-\mathrm{C} 23-\mathrm{C} 32$ & $-0.7(4)$ \\
\hline $\mathrm{O} 3-\mathrm{C} 4-\mathrm{C} 5-\mathrm{C} 6$ & $0.4(4)$ & $\mathrm{O} 8-\mathrm{C} 22-\mathrm{C} 23-\mathrm{C} 24$ & $-1.7(4)$ \\
\hline $\mathrm{C} 3-\mathrm{C} 4-\mathrm{C} 5-\mathrm{C} 6$ & $-179.4(2)$ & $\mathrm{C} 21-\mathrm{C} 22-\mathrm{C} 23-\mathrm{C} 24$ & $179.4(3)$ \\
\hline $\mathrm{C} 4-\mathrm{C} 5-\mathrm{C} 6-\mathrm{O} 4$ & $-0.1(4)$ & $\mathrm{C} 22-\mathrm{C} 23-\mathrm{C} 24-\mathrm{O} 9$ & $-0.4(4)$ \\
\hline $\mathrm{C} 14-\mathrm{C} 5-\mathrm{C} 6-\mathrm{O} 4$ & $-178.5(3)$ & $\mathrm{C} 32-\mathrm{C} 23-\mathrm{C} 24-\mathrm{O} 9$ & $179.7(3)$ \\
\hline $\mathrm{C} 4-\mathrm{C} 5-\mathrm{C} 6-\mathrm{C} 7$ & $179.8(2)$ & $\mathrm{C} 22-\mathrm{C} 23-\mathrm{C} 24-\mathrm{C} 25$ & $178.4(3)$ \\
\hline $\mathrm{C} 14-\mathrm{C} 5-\mathrm{C} 6-\mathrm{C} 7$ & $1.4(4)$ & $\mathrm{C} 32-\mathrm{C} 23-\mathrm{C} 24-\mathrm{C} 25$ & $-1.5(4)$ \\
\hline
\end{tabular}




$\begin{array}{ll}\mathrm{O} 4-\mathrm{C} 6-\mathrm{C} 7-\mathrm{C} 8 & -0.8(4) \\ \mathrm{C} 5-\mathrm{C} 6-\mathrm{C} 7-\mathrm{C} 8 & 179.3(2) \\ \mathrm{O} 4-\mathrm{C} 6-\mathrm{C} 7-\mathrm{C} 12 & 178.8(3) \\ \mathrm{C} 5-\mathrm{C} 6-\mathrm{C} 7-\mathrm{C} 12 & -1.0(4) \\ \mathrm{C} 12-\mathrm{C} 7-\mathrm{C} 8-\mathrm{C} 9 & 0.1(4) \\ \mathrm{C} 6-\mathrm{C} 7-\mathrm{C} 8-\mathrm{C} 9 & 179.8(3) \\ \mathrm{C} 7-\mathrm{C} 8-\mathrm{C} 9-\mathrm{C} 10 & -0.8(4) \\ \mathrm{C} 8-\mathrm{C} 9-\mathrm{C} 10-\mathrm{C} 11 & 1.5(4) \\ \mathrm{C} 9-\mathrm{C} 10-\mathrm{C} 11-\mathrm{C} 12 & -1.6(4) \\ \mathrm{C} 8-\mathrm{C} 7-\mathrm{C} 12-\mathrm{C} 11 & -0.2(4) \\ \mathrm{C} 6-\mathrm{C} 7-\mathrm{C} 12-\mathrm{C} 11 & -179.9(2) \\ \mathrm{C} 8-\mathrm{C} 7-\mathrm{C} 12-\mathrm{C} 13 & 179.3(2) \\ \mathrm{C} 6-\mathrm{C} 7-\mathrm{C} 12-\mathrm{C} 13 & -0.3(4) \\ \mathrm{C} 10-\mathrm{C} 11-\mathrm{C} 12-\mathrm{C} 7 & 1.0(4) \\ \mathrm{C} 10-\mathrm{C} 11-\mathrm{C} 12-\mathrm{C} 13 & -178.6(2) \\ \mathrm{C} 7-\mathrm{C} 12-\mathrm{C} 13-\mathrm{O} 5 & -178.4(3) \\ \mathrm{C} 11-\mathrm{C} 12-\mathrm{C} 13-\mathrm{O} 5 & 1.2(4) \\ \mathrm{C} 7-\mathrm{C} 12-\mathrm{C} 13-\mathrm{C} 14 & 1.3(4) \\ \mathrm{C} 11-\mathrm{C} 12-\mathrm{C} 13-\mathrm{C} 14 & -179.1(2) \\ \mathrm{O} 1-\mathrm{C} 1-\mathrm{C} 14-\mathrm{C} 5 & 179.7(2) \\ \mathrm{C} 2-\mathrm{C} 1-\mathrm{C} 14-\mathrm{C} 5 & 0.4(4) \\ \mathrm{O} 1-\mathrm{C} 1-\mathrm{C} 14-\mathrm{C} 13 & -1.0(4) \\ \mathrm{C} 2-\mathrm{C} 1-\mathrm{C} 14-\mathrm{C} 13 & 179.6(2) \\ \mathrm{C} 4-\mathrm{C} 5-\mathrm{C} 14-\mathrm{C} 1 & 0.5(4) \\ \mathrm{C} 6-\mathrm{C} 5-\mathrm{C} 14-\mathrm{C} 1 & 178.9(2) \\ \mathrm{C} 4-\mathrm{C} 5-\mathrm{C} 14-\mathrm{C} 13 & -178.8(2) \\ \mathrm{C} 6-\mathrm{C} 5-\mathrm{C} 14-\mathrm{C} 13 & -0.4(4) \\ \mathrm{O} 5-\mathrm{C} 13-\mathrm{C} 14-\mathrm{C} 1 & -0.6(4) \\ \mathrm{C} 12-\mathrm{C} 13-\mathrm{C} 14-\mathrm{C} 1 & 179.7(2) \\ \mathrm{O} 5-\mathrm{C} 13-\mathrm{C} 14-\mathrm{C} 5 & 178.7(3) \\ \mathrm{C} 12-\mathrm{C} 13-\mathrm{C} 14-\mathrm{C} 5 & -1.0(4) \\ \mathrm{C} 2-\mathrm{O} 2-\mathrm{C} 15-\mathrm{C} 16 & -65.1(3) \\ \mathrm{O} 2-\mathrm{C} 15-\mathrm{C} 16-\mathrm{C} 17 & -173.1(2) \\ \mathrm{C} 15-\mathrm{C} 16-\mathrm{C} 17-\mathrm{C} 18 & \\ & \end{array}$

$\begin{array}{ll}\mathrm{O} 9-\mathrm{C} 24-\mathrm{C} 25-\mathrm{C} 26 & -0.3(4) \\ \mathrm{C} 23-\mathrm{C} 24-\mathrm{C} 25-\mathrm{C} 26 & -179.1(3) \\ \mathrm{O} 9-\mathrm{C} 24-\mathrm{C} 25-\mathrm{C} 30 & 179.0(3) \\ \mathrm{C} 23-\mathrm{C} 24-\mathrm{C} 25-\mathrm{C} 30 & 0.2(4) \\ \mathrm{C} 30-\mathrm{C} 25-\mathrm{C} 26-\mathrm{C} 27 & -1.4(4) \\ \mathrm{C} 24-\mathrm{C} 25-\mathrm{C} 26-\mathrm{C} 27 & 178.0(3) \\ \mathrm{C} 25-\mathrm{C} 26-\mathrm{C} 27-\mathrm{C} 28 & 1.6(5) \\ \mathrm{C} 26-\mathrm{C} 27-\mathrm{C} 28-\mathrm{C} 29 & -0.9(5) \\ \mathrm{C} 27-\mathrm{C} 28-\mathrm{C} 29-\mathrm{C} 30 & 0.1(4) \\ \mathrm{C} 28-\mathrm{C} 29-\mathrm{C} 30-\mathrm{C} 25 & 0.1(4) \\ \mathrm{C} 28-\mathrm{C} 29-\mathrm{C} 30-\mathrm{C} 31 & 178.8(3) \\ \mathrm{C} 26-\mathrm{C} 25-\mathrm{C} 30-\mathrm{C} 29 & 0.6(4) \\ \mathrm{C} 24-\mathrm{C} 25-\mathrm{C} 30-\mathrm{C} 29 & -178.8(3) \\ \mathrm{C} 26-\mathrm{C} 25-\mathrm{C} 30-\mathrm{C} 31 & -178.2(3) \\ \mathrm{C} 24-\mathrm{C} 25-\mathrm{C} 30-\mathrm{C} 31 & 2.5(4) \\ \mathrm{C} 29-\mathrm{C} 30-\mathrm{C} 31-\mathrm{O} 10 & -2.8(4) \\ \mathrm{C} 25-\mathrm{C} 30-\mathrm{C} 31-\mathrm{O} 10 & 176.0(2) \\ \mathrm{C} 29-\mathrm{C} 30-\mathrm{C} 31-\mathrm{C} 32 & 177.3(3) \\ \mathrm{C} 25-\mathrm{C} 30-\mathrm{C} 31-\mathrm{C} 32 & -3.9(4) \\ \mathrm{O} 6-\mathrm{C} 19-\mathrm{C} 32-\mathrm{C} 23 & 178.8(2) \\ \mathrm{C} 20-\mathrm{C} 19-\mathrm{C} 32-\mathrm{C} 23 & -0.5(4) \\ \mathrm{O} 6-\mathrm{C} 19-\mathrm{C} 32-\mathrm{C} 31 & -0.9(4) \\ \mathrm{C} 20-\mathrm{C} 19-\mathrm{C} 32-\mathrm{C} 31 & 179.8(2) \\ \mathrm{C} 22-\mathrm{C} 23-\mathrm{C} 32-\mathrm{C} 19 & 0.5(4) \\ \mathrm{C} 24-\mathrm{C} 23-\mathrm{C} 32-\mathrm{C} 19 & -179.6(3) \\ \mathrm{C} 22-\mathrm{C} 23-\mathrm{C} 32-\mathrm{C} 31 & -179.9(2) \\ \mathrm{C} 24-\mathrm{C} 23-\mathrm{C} 32-\mathrm{C} 31 & 0.0(4) \\ \mathrm{O} 10-\mathrm{C} 31-\mathrm{C} 32-\mathrm{C} 19 & 2.4(4) \\ \mathrm{C} 30-\mathrm{C} 31-\mathrm{C} 32-\mathrm{C} 19 & -177.7(2) \\ \mathrm{O} 10-\mathrm{C} 31-\mathrm{C} 32-\mathrm{C} 23 & -177.2(2) \\ \mathrm{C} 30-\mathrm{C} 31-\mathrm{C} 32-\mathrm{C} 23 & 2.7(4) \\ \mathrm{C} 20-\mathrm{O} 7-\mathrm{C} 33-\mathrm{C} 34 & -167.3(2) \\ \mathrm{O} 7-\mathrm{C} 33-\mathrm{C} 34-\mathrm{C} 35 & -174.3(3) \\ \mathrm{C} 33-\mathrm{C} 34-\mathrm{C} 35-\mathrm{C} 36 & -175.2(3) \\ & \end{array}$

Hydrogen-bond geometry $\left(A,{ }^{\circ}\right)$

\begin{tabular}{lllll}
\hline$D-\mathrm{H} \cdots A$ & $D-\mathrm{H}$ & $\mathrm{H} \cdots A$ & $D \cdots A$ & $D-\mathrm{H} \cdots A$ \\
\hline $\mathrm{O} 1-\mathrm{H} 1 O \cdots \mathrm{O} 5$ & $0.85(2)$ & $1.78(2)$ & $2.564(3)$ & $153(3)$ \\
$\mathrm{O} 3-\mathrm{H} 3 O \cdots \mathrm{O} 4$ & $0.87(2)$ & $1.74(2)$ & $2.536(3)$ & $151(3)$ \\
$\mathrm{O} 6-\mathrm{H} 6 O \cdots \mathrm{O} 10$ & $0.86(2)$ & $1.72(2)$ & $2.542(3)$ & $158(3)$ \\
$\mathrm{O} 8-\mathrm{H} 8 O \cdots \mathrm{O} 9$ & $0.87(2)$ & $1.73(2)$ & $2.554(3)$ & $155(3)$ \\
$\mathrm{C} 3-\mathrm{H} 3 \cdots \mathrm{O} 10$ & 0.95 & 2.55 & $3.494(3)$ & 173 \\
$\mathrm{C} 29-\mathrm{H} 29 \cdots \mathrm{O} 3$ & 0.95 & 2.41 & $3.231(4)$ & 144 \\
$\mathrm{C} 15-\mathrm{H} 15 B \cdots \mathrm{O}^{\mathrm{i}}$ & 0.99 & 2.52 & $3.485(3)$ & 164
\end{tabular}


supporting information

\begin{tabular}{llllr}
$\mathrm{C} 21-\mathrm{H} 21 \cdots \mathrm{O} 8^{\mathrm{ii}}$ & 0.95 & 2.55 & $3.502(3)$ & 180 \\
$\mathrm{C} 33-\mathrm{H} 33 A \cdots \mathrm{O} 9^{\mathrm{iii}}$ & 0.99 & 2.56 & $3.547(4)$ & 172 \\
\hline
\end{tabular}

Symmetry codes: (i) $x-1, y, z$; (ii) $-x+3,-y+1,-z+1$; (iii) $-x+2,-y+1,-z+1$. 